\title{
ARHGEF4 predicts poor prognosis and promotes cell invasion by influencing ERK1/2 and GSK-3 $\alpha / \beta$ signaling in pancreatic cancer
}

\author{
KEISUKE TANIUCHI $^{1,2}$, MUTSUO FURIHATA ${ }^{3}$, SEIJI NAGANUMA ${ }^{3}$ and TOSHIJI SAIBARA S $^{1,2}$ \\ Departments of ${ }^{1}$ Endoscopic Diagnostics and Therapeutics, ${ }^{2}$ Gastroenterology and Hepatology, and \\ ${ }^{3}$ Pathology, Kochi Medical School Hospital, Kochi University, Nankoku, Kochi 783-8505, Japan
}

Received January 31, 2018; Accepted July 5, 2018

DOI: 10.3892/ijo.2018.4549

\begin{abstract}
Rho guanine nucleotide exchange factor 4 (ARHGEF4) is a guanine nucleotide exchange factor that is specific for Rac1 and Cdc42. The aim of the present study was to investigate the role of ARHGEF4 in the motility and invasiveness of pancreatic cancer cells. Evaluation of an immunohistochemical staining of 102 resected pancreatic cancer samples demonstrated that high ARHGEF4 expression was correlated with an independent predictor of worse overall survival in univariate and multivariate analyses. Immunofluorescence analyses and Matrigel invasion assays demonstrated that suppression of ARHGEF4 inhibited the formation of membrane protrusions, and in turn inhibited cell motility and invasion. A phosphoprotein array analysis demonstrated that knockdown of ARHGEF4 decreased phosphorylated extracellular signal-regulated kinase (ERK)1/2 and glycogen synthase kinase-3 (GSK-3) $\alpha / \beta$ in pancreatic cancer cells, and ERK1/2 and GSK-3 $\alpha / \beta$ were associated with ARHGEF4-related motility and invasiveness through an increase in cell protrusions. These results suggested that ARHGEF4 stimulates ERK1/2 and GSK-3 $\alpha / \beta$, and provided evidence that ARHGEF4 promotes cell motility and invasiveness. Inhibition of ARHGEF4 may be a novel approach to a targeted molecular therapy, as any such therapy would limit the motility and invasiveness of pancreatic cancer cells.
\end{abstract}

\section{Introduction}

Rho guanine nucleotide exchange factor 4 (ARHGEF4), which is also referred to as ASEF, has been identified as a Rac1-specific guanine nucleotide exchange factor (GEF) that interacts with adenomatous polyposis coli (APC) (1). ARHGEF4 is a Dbl family GEF that contains an Src homology 3 (SH3) domain

Correspondence to: Dr Keisuke Taniuchi, Department of Endoscopic Diagnostics and Therapeutics, Kochi Medical School Hospital, Kochi University, 185-1 Kohasu, Oko-cho, Nankoku, Kochi 783-8505, Japan

E-mail: ktaniuchi@kochi-u.ac.jp

Key words: guanine nucleotide exchange factor, pancreatic cancer, cell invasion, cell protrusion, actin cytoskeleton followed by the Dbl homology and pleckstrin homology domains that are characteristic of Dbl family GEFs, which specifically activate members of the Rho family of GTPases (2). APC binds to the $\mathrm{NH}_{2}$-terminal APC-binding region of ARHGEF4 via its armadillo repeat domain and enhances the GEF activity of ARHGEF4 against Rac1 and Cdc42, thereby regulating the reorganization of the actin cytoskeleton, cell morphology, adhesion, and migration (3-5). Truncated mutant APC present in colorectal cancer cells strongly enhance the constitutive GEF activity of ARHGEF4, which upregulates the expression of matrix metalloproteinase 9 via the c-Jun $\mathrm{NH}_{2}$-terminal kinase (JNK) pathway, and causes decreased cell-cell adhesion and aberrant migratory properties (6-8). Collectively, these reports demonstrated that ARHGEF4 is associated with motility and invasiveness via its GEF activity.

The majority of patients with pancreatic ductal adenocarcinoma (PDAC) progress to either locally invasive or metastatic disease (9); however, the detailed mechanism by which PDAC cells invade and metastasize remains unknown. The present authors previously reported that insulin-like growth factor-2 mRNA-binding protein 3 (IGF2BP3) and IGF2BP3-bound mRNAs are localized to cytoplasmic RNA granules that accumulate in cell protrusions of PDAC cells $(10,11)$. IGF2BP3-bound transcripts, including ARHGEF4, are preferentially translated in cell protrusions and induce the formation of cell protrusions; consequently, IGF2BP3 promotes invasiveness and metastasis (10). These findings indicate that IGF2BP3 contributes to translational regulation only in the cell protrusions and that ARHGEF4 translated in the protrusions is associated with cell invasion and metastasis.

The present study aimed to examine the ARHGEF4 expression in tissue samples of PDAC patients and its association with clinicopathologic characteristics and survival, and to evaluate the mechanism of ARHGEF4 in the control of PDAC cell motility and invasion. It was demonstrated that ARHGEF4 induces the formation of cell protrusions by increasing phosphorylated extracellular signal-regulated kinase (ERK)1/2 and glycogen synthase kinase-3 (GSK-3) $\alpha / \beta$, resulting in an increase in motility and invasiveness in PDAC cells.

\section{Materials and methods}

Antibodies. Anti-ARHGEF4 (55213-1-AP) antibody was purchased from Proteintech Group, Inc. (Chicago, IL, USA). 
Anti-GSK-3 $\alpha / \beta$ (sc7291), anti-myc (sc40) and anti-GAPDH (sc32233) antibodies were purchased from Santa Cruz Biotechnology, Inc. (Dallas, TX, USA). Anti-ERK1/2 (4697) and anti-phosphorylated ERK1/2 (Thr204/Tyr187; 5726) antibodies were purchased from Cell Signaling Technology, Inc. (Danvers, MA, USA). Anti-phosphorylated GSK-3 $\alpha$ (Ser9; 75814) and anti-phosphorylated GSK-3 $\beta$ (Tyr216; 75745) antibodies were purchased from Abcam (Cambridge, MA, USA). Anti-caspase-8 antibody (LS-C344789) was purchased from LifeSpan BioSciences, Inc. (Seattle, WA, USA). The JLA20 anti-actin antibody (MABT219) was purchased from Merck KGaA (Darmstadt, Germany).

Primary human PDAC samples. Tumor tissues were obtained from 102 patients who underwent surgical treatment for pancreatic intraepithelial neoplasm (PanIN, n=2) and PDAC $(n=100)$ and received surgical resection during 1999-2014 at the Departments of Surgery at Kochi Medical School Hospital (Nankoku, Japan) and Matsuyama Shimin Hospital (Matsuyama, Japan), as published previously (12). Postoperative follow-up consisted of physical examination, measurement of serum sialyl Lewis (a) blood group antigen (CA19-9), which is the clinical standard PDAC tumor biomarker, and computed tomography at 3-4-month intervals at Kochi Medical School Hospital (Nankoku, Japan) and Matsuyama Shimin Hospital (Matsuyama, Japan). The disease status of each patient was determined at the date of the final follow up in 2014. If patients succumbed during follow-up, PanIN- or PDAC-associated death was considered an outcome event. Medical records of the 102 patients provided information regarding sex, age, tumor diameter, histology, Union for International Cancer Control (UICC) tumor-node-metastasis (TNM) stage, venous invasion, lymphatic invasion and postoperative survival time. Tumors were classified according to the classification of pancreatic carcinoma of the Japan Pancreas Society (JPS) (13) and the UICC TNM classification (14). The JPS staging system is adequate for analysis of resected PDAC cases, and the UICC staging system is more suitable for analyses of both resected and non-resected PDAC patients (15). It is recommended to record the clinical and pathological information according to both JPS and UICC staging systems (15). The present study was approved by the Ethical Review Board of Kochi Medical School and Matsuyama Shimin Hospital prior to patient recruitment. In conformity with the principles of the Declaration of Helsinki, written informed consent was acquired from each patient prior to initiation.

Immunohistochemical staining. Immunohistochemistry was carried out, as published previously (12). Tissue sections from normal pancreas, brain, lung, liver and kidney were purchased from BioChain Institute, Inc. (Hayward, CA, USA).

Evaluation of ARHGEF4 staining. A total of 10 random microscopic fields (original magnification, $x 400$ ) per slide were evaluated by two independent observers (SN and MF) who were blinded to clinical and outcome data. ARHGEF4 expression levels were classified semi-quantitatively based on the total combined scores of positive-staining tumor cell percentage $(1,<50 \%$ reacting cells; $2,50-80 \%$ reacting cells; $3,>80 \%)$ and staining intensity (1, weaker than the intensity of surface staining in the islet of Langerhans; 2, equal to the intensity of the islet of Langerhans; 3, stronger than the intensity of the islet of Langerhans). A total immunohistochemical score was calculated by summing the percentage score and the intensity score. ARHGEF4 expression was classified into two groups based on total score (low group, 2-3; high group, 4-6) in accordance with previous studies $(12,16)$.

Cell culture. The human PDAC cell line S2-013, a subline of SUIT-2, was obtained from Dr T. Iwamura (Miyazaki Medical College, Miyazaki, Japan) (17). The human PDAC cell line PANC-1 was purchased from the American Type Culture Collection (Manassas, VA, USA). All cells were maintained in Dulbecco's modified Eagle's medium (Gibco; Thermo Fisher Scientific, Inc., Waltham, MA, USA) containing $10 \%$ fetal calf serum (FCS; Gibco, Thermo Fisher Scientific, Inc.) at $37^{\circ} \mathrm{C}$. In selected experiments, cells were incubated at $37^{\circ} \mathrm{C}$ with $30 \mu \mathrm{M}$ U0126 (Sigma-Aldrich; Merck KGaA), a specific mitogenactivated protein kinase kinase (MEK) 1/2 inhibitor, for $24 \mathrm{~h}$ or with $20 \mu \mathrm{M}$ SB216763 (Sigma-Aldrich; Merck KGaA), a specific GSK-3 inhibitor, for $48 \mathrm{~h}$.

Confocal immunofluorescence microscopy. Immunocytochemistry was performed as previously detailed $(18,19)$. Briefly, S2-013 and PANC-1 cells incubated on fibronectincoated glass coverslips for $5 \mathrm{~h}$ at $37^{\circ} \mathrm{C}$ were fixed with $4 \%$ paraformaldehyde for $30 \mathrm{~min}$ and permeabilized with $0.1 \%$ Triton X-100 for $2 \mathrm{~min}$ at room temperature. Cells were then incubated with anti-ARHGEF4, anti-myc, anti-phosphorylated ERK1/2 or anti-caspase- 8 primary antibodies (all 1:100) for $1 \mathrm{~h}$ at room temperature, followed by incubation with Alexa488- or Alexa594-conjugated secondary antibodies at 1:500 for $1 \mathrm{~h}$ at room temperature (A32723 and R37117, respectively; Molecular Probes, Thermo Fisher Scientific, Inc.). Each specimen was visualized using a Zeiss LSM 510 META microscope (Carl Zeiss AG, Oberkochen, Germany) at X80 magnification. Z-stacks of confocal images (approximately 10 stacks/cell) were taken using a Zeiss LSM 510 META microscope at X80 magnification.

S2-013 and PANC-1 cells that formed cell protrusions were counted by two blinded individuals (SN and TS). Four independent visual fields were counted via microscopic observation to count the number of cells that formed cell protrusions. Data are derived from three independent experiments.

Small interfering (si)RNA treatment. A single mixture with four different small interfering RNA (siRNA) oligonucleotides targeting ARHGEF4 (FlexiTube GeneSolution GS50649) was purchased from Qiagen, Inc. (Valencia, CA, USA) and a single mixture with four different scrambled negative control siRNA oligonucleotides (37007) was obtained from Santa Cruz Biotechnology, Inc.; S2-013 and PANC-1 cells were transfected with 80 pmol of each siRNA mixture in siRNA transfection reagent (31985-062; HiPerfect Transfection Reagent; Qiagen, Inc.) following the manufacturer's protocol. Following incubation for $48 \mathrm{~h}$ at $37^{\circ} \mathrm{C}$, total cell lysates were extracted, and immunoblotting was carried out to evaluate the effects of siRNA treatment.

ARHGEF4-rescue construct. Total RNA were extracted from S2-013 cells using TRIzol reagent (Invitrogen; Thermo Fisher 
Scientific, Inc.) according to the manufacturer's recommendations, and reverse transcribed to single-stranded cDNAs using SuperScript III First-Strand Synthesis system for RT-PCR (18080-051; Invitrogen, Thermo Fisher Scientific, Inc.) and oligo(dT $)_{12-18}$ primer for $50 \mathrm{~min}$ at $50^{\circ} \mathrm{C}$. Polymerase chain reaction (PCR) amplification was performed using an AmpliTaq Gold DNA Polymerase kit (N8080241; Invitrogen, Thermo Fisher Scientific, Inc.) and the primer sequences for ARHGEF4 were forward, 5'-CCAAGCTTATGCCCTGGGAAGAAC CAGC-3' and reverse, 5'-CCGCTCGAGTTGCTGGCTTG GCTTTGTGG-3'. All reactions were comprised of initial denaturation at $94^{\circ} \mathrm{C}$ for 2 min followed by 28 cycles at $94^{\circ} \mathrm{C}$ for $30 \mathrm{sec}, 58^{\circ} \mathrm{C}$ for $30 \mathrm{sec}$, and $72^{\circ} \mathrm{C}$ for $1 \mathrm{~min}$, on a Takara PCR Thermal Cycler Dice (Takara Bio, Inc., Otsu, Japan). The product was then inserted into the HindIII and XhoI sites of pCMV6-Entry vector (Origene Technologies, Inc., Rockville, MD, USA) bearing a C-terminal myc-DDK-tag. Transient transfection of resultant ARHGEF4-rescue construct was carried out with X-tremeGENE HP DNA transfection reagent (Roche Applied Science, Penzberg, Germany) at room temperature. The transfected cells were assayed, typically 2 days following transfection.

Immunoblotting analysis of cell lysates. Total cell lysates were extracted using lysis buffer [Tris- $\mathrm{HCl}(\mathrm{pH} 7.4)$, sodium dodecyl sulfate (SDS), mercaptoethanol and glycerol]. Protein concentrations were determined using Takara Bradford Protein Assay kit (T9310A; Takara Bio, Inc.), and an equal amount of protein $(10 \mu \mathrm{g})$ was separated on a $4-20 \%$ gradient SDS-PAGE (TEFCO, Tokyo, Japan). Proteins were transferred using a Trans-Blot Turbo RTA Mini LF PVDF Transfer kit (170-4274) to a TransBlot Turbo Mini-size LF polyvinylidene difluoride membrane (both from Bio-Rad Laboratories, Inc., Hercules, CA, USA) and blocked with 5\% non-fat dry milk in Tris-buffered saline [10 mM Tris- $\mathrm{HCl}$ (pH 7.4), $150 \mathrm{mM} \mathrm{NaCl}$, $0.1 \%$ Tween-20] for $1 \mathrm{~h}$ at room temperature. The membranes were then incubated with anti-ARHGEF4, anti-myc, antiERK1/2, anti-phosphorylated ERK1/2, anti-GSK-3 $\alpha / \beta$, anti-phosphorylated GSK-3 $\alpha$, anti-phosphorylated GSK-3 $\beta$, anti-caspase- 8 or anti-GAPDH primary antibodies at dilutions of 1:1,000 in 5\% non-fat dry milk in Tris-buffered saline overnight at $4^{\circ} \mathrm{C}$. Following incubation with appropriate secondary antibodies conjugated with horseradish peroxidase (sc2004, sc2005; Santa Cruz Biotechnology, Inc.) at dilutions of 1:2,000 for $1 \mathrm{~h}$ at room temperature, the immunoreactive bands were visualized using the ECL Plus kit (GE Healthcare, Chicago, IL, USA) according to the manufacturer's instructions.

Transwell motility assay. A Transwell motility assay was carried out, as detailed previously (10). Cells $\left(3.0 \times 10^{4}\right)$ were added to the upper chamber of BD BioCoat Control Culture inserts (24-well plates, $8-\mu \mathrm{m}$ pore size; BD Biosciences, San Jose, CA, USA). A total of $200 \mu 1$ serum-free Dulbecco's modified Eagle's medium (Gibco; Thermo Fisher Scientific, Inc.) was added to the top well and $700 \mu \mathrm{l}$ medium containing $5 \%$ FCS to the bottom well. Following incubation for $12 \mathrm{~h}$ at $37^{\circ} \mathrm{C}$, the bound cells were stained with $0.1 \%$ crystal violet solution for $10 \mathrm{~min}$ at room temperature, and four independent visual fields were examined via light microscopy (BX41; Olympus Corporation, Tokyo, Japan) at x100 magnification to count the number of cells that had migrated to the bottom chamber. The assay was conducted three times independently.

Matrigel invasion assay. A Matrigel invasion assay was carried out, as published previously (10). The invasion capabilities of PDAC cells were examined using a two-chamber invasion assay kit (24-well plates, $8-\mu \mathrm{m}$ pore size membrane coated with a layer of Matrigel extracellular matrix proteins; BD Biosciences) according to the manufacturer's instructions. The bound cells were stained with $0.1 \%$ crystal violet solution for $10 \mathrm{~min}$ at room temperature, and four independent visual fields were examined via light microscopy (BX41; Olympus Corporation) at x100 magnification to count the number of cells that had moved to the bottom chamber. The assay was conducted three times independently.

Phospho-kinase array assay. The Proteome Profiler Human Phospho-Kinase Array kit (ARY003) was purchased from R\&D Systems, Inc. (Minneapolis, MN, USA) and screened according to the manufacturer's protocol, as published previously (20).

Subcellular fractionation. S2-013 and PANC-1 cells were treated with $100 \mu \mathrm{M}$ cytochalasin D (22144-77-0; Fermentek, Ltd., Jerusalem, Israel) for $12 \mathrm{~h}$ at $37^{\circ} \mathrm{C}$, and then incubated on fibronectin for $5 \mathrm{~h}$ at $37^{\circ} \mathrm{C}$. The cells were extracted for $10 \mathrm{~min}$ on ice in lysis buffer [100 mM PIPES (pH 6.8), $1 \mathrm{mM}$ EGTA, $1 \mathrm{mM} \mathrm{MgCl} 2$, $1 \%$ Triton X-100 (Nacalai Tesque, Inc., Kyoto, Japan)] containing $2 \mu \mathrm{M}$ phalloidin, protease inhibitor cocktail tablets (Roche Applied Science), and phosphatase inhibitor cocktail (Nacalai Tesque, Inc.). Following centrifugation at $10,000 \times \mathrm{g}$ for $5 \mathrm{~min}$ at $4^{\circ} \mathrm{C}$, the soluble fraction was removed, and the residual insoluble fraction was washed once with lysis buffer and scraped with sample buffer $[50 \mathrm{mM}$ Tris-HCL ( $\mathrm{pH}$ 6.8), 2\% SDS, $0.02 \%$ bromophenol blue, $1 \% \beta$-mercaptoethanol and $10 \%$ glycerol]. Equal amounts of soluble and insoluble fractions $(10 \mu \mathrm{g})$ were subjected to a 4-20\% gradient SDS-PAGE (TEFCO) and analyzed by immunoblotting using anti-ARHGEF4 and anti-actin antibodies with dilution at 1:1,000.

Statistical analysis. For immunohistochemical analysis, statistical analyses were performed using R (version 3.3.3; The R Foundation, Wien, Austria), as published previously (12). Cumulative survival rates were calculated using the Kaplan-Meier method and were compared using the logrank test (Mantel-Cox). Survival rates are expressed as the median value and interquartile range (IQR). Univariate Cox regression analysis was performed to determine the prognostic significance of individual clinicopathological factors. Cox proportional hazard models were used for multivariate analysis of independent factors for overall survival. For the in vitro experiments, statistical significance was evaluated by using Student's t-test. $\mathrm{P}<0.05$ was considered to indicate a statistically significant difference.

\section{Results}

ARHGEF4 expression in PDAC samples. The potential significance of ARHGEF4 protein expression was examined 
in surgical specimens from 102 patients with PanIN $(n=2)$ and PDAC $(n=100)$ by immunohistochemical analysis. PDAC is thought to arise from precursor lesions, PanIN (21). ARHGEF4 demonstrated positive immunoreactivity in all 102 cases, and the specimens were classified into low-expressing (62.7\%) and highexpressing (37.3\%) ARHGEF4 groups (Table I). ARHGEF4 was localized in the cytoplasm of PDAC cells (Fig. 1A), whereas ARHGEF4 was low-expressing in PanIN cells, indicating that PanINs do not contain ARHGEF4 (Fig. 1B). The level of ARHGEF4 expression at the invasive front of the tumor was not increased when compared with the central region of the tumor (Fig. 1C). ARHGEF4 immunoreactivity was not observed in normal pancreatic ducts (Fig. 1D). In addition, normal brain, lung, liver and kidney were not stained by the anti-ARHGEF4 antibody (Fig. 1E).

Association among ARHGEF4 expression, clinicopathological characteristics and survival. The associations between ARHGEF4 expression and clinicopathological factors are presented in Table II. No significant associations were noted between ARHGEF4 expression and any clinicopathological parameters.

Subsequently, the correlation between ARHGEF4 expression and PDAC patient prognosis was examined. The follow-up period for the 102 PDAC survivors ranged from 18-192 months (median, 64.0 months). The overall survival time for PDAC patients with high ARHGEF4 expression was significantly shorter than that of PDAC patients with low ARHGEF4 expression on Kaplan-Meier curves $(\mathrm{P}<0.001$; Fig. 2$)$. In relation to clinical outcome, univariate and multivariate analyses revealed that stage III and IV and high ARHGEF4 expression were independent predictors of worse overall survival (Table III). These results suggested that ARHGEF4 participated in PDAC progression.

Subcellular localization of ARHGEF4 in PDAC cells. Immunocytochemistry was used to investigate the subcellular localization of ARHGEF4 in moderately differentiated PDAC cells (line S2-013) (17) and cells from a poorly differentiated PDAC line (PANC-1) (22). Fibronectin induces the formation of cell protrusions at the leading edge of PDAC cells $(23,24)$. There were fewer cell protrusions formed by S2-013 and PANC-1 cells when the cells were cultured without fibronectin than when the cells are grown on fibronectin $(12,20)$. In S2-013 and PANC-1 cells grown on fibronectin, ARHGEF4 was mainly present in the cytoplasm of cell bodies, and ARHGEF4 was also localized in cell protrusions containing many peripheral actin structures (Fig. 3A). ARHGEF4, which accumulates in cell protrusions, accumulated in abundance in the S2-013 and PANC-1 cells cultured on fibronectin compared with the corresponding cells that were not cultured on fibronectin (Fig. 3A). S2-013 cells grown on fibronectin exhibited intracellular expression of ARHGEF4 in cell protrusions in Z stack panels (Fig. 3B).

Localization of ARHGEF4 in cell protrusions. To quantify the subcellular distribution of ARHGEF4 in S2-013 and PANC-1 cells cultured with or without fibronectin, cell lysates were fractionated into detergent-soluble and -insoluble (cytoskeletal) fractions. The amounts of ARHGEF4 and actin in the
Table I. Summary of characteristics in 102 patients with pancreatic cancer.

\begin{tabular}{|c|c|c|}
\hline Characteristic & Patients, n & $\%$ of tota \\
\hline \multicolumn{3}{|c|}{ Age at surgery, years } \\
\hline $40-50$ & 4 & 3.9 \\
\hline $50-60$ & 17 & 16.7 \\
\hline $60-70$ & 32 & 31.4 \\
\hline $70-80$ & 41 & 40.2 \\
\hline$>80$ & 8 & 7.8 \\
\hline \multicolumn{3}{|l|}{ Sex } \\
\hline Male & 56 & 54.9 \\
\hline Female & 46 & 45.1 \\
\hline \multicolumn{3}{|l|}{ Stage $^{a}$} \\
\hline 0 & 2 & 2.0 \\
\hline IA & 4 & 3.9 \\
\hline IB & 8 & 7.8 \\
\hline IIA & 32 & 31.4 \\
\hline IIB & 50 & 49.0 \\
\hline III & 2 & 2.0 \\
\hline IV & 4 & 3.9 \\
\hline \multicolumn{3}{|l|}{ Primary tumor ${ }^{\mathrm{a}}$} \\
\hline Tis & 2 & 2.0 \\
\hline $\mathrm{T} 1$ & 6 & 5.9 \\
\hline $\mathrm{T} 2$ & 15 & 14.6 \\
\hline T3 & 77 & 75.5 \\
\hline $\mathrm{T} 4$ & 2 & 2.0 \\
\hline \multicolumn{3}{|c|}{ Regional lymph nodes ${ }^{\mathrm{a}}$} \\
\hline N0 & 46 & 45.1 \\
\hline $\mathrm{N} 1$ & 56 & 54.9 \\
\hline \multicolumn{3}{|c|}{ Distant metastasis ${ }^{\mathrm{a}}$} \\
\hline M0 & 98 & 96.1 \\
\hline M1 & 4 & 3.9 \\
\hline \multicolumn{3}{|l|}{ Histologyb } \\
\hline PanIN & 2 & 2.0 \\
\hline Well & 31 & 30.4 \\
\hline Moderate & 57 & 55.8 \\
\hline Poor & 12 & 11.8 \\
\hline \multicolumn{3}{|c|}{ Venous invasion $^{\mathrm{b}}$} \\
\hline v0 & 57 & 55.4 \\
\hline $\mathrm{v} 1$ & 31 & 30.7 \\
\hline $\mathrm{v} 2$ & 11 & 10.9 \\
\hline v3 & 3 & 3.0 \\
\hline \multicolumn{3}{|c|}{ Lymphatic invasion $^{\mathrm{b}}$} \\
\hline ly0 & 43 & 42.6 \\
\hline ly1 & 34 & 33.6 \\
\hline ly2 & 21 & 19.9 \\
\hline $\operatorname{ly} 3$ & 4 & 3.9 \\
\hline \multicolumn{3}{|c|}{ ARHGEF4 expression } \\
\hline Low & 64 & 62.7 \\
\hline High & 38 & 37.3 \\
\hline
\end{tabular}

${ }^{a}$ Classified according to the classification of International Union against Cancer; ${ }^{b}$ classified according to the classification of pancreatic cancer of Japan Pancreas Society. Tis, intraepithelial carcinoma; PanIN, pancreatic intraepithelial neoplasia; ARHGEF4, rho guanine nucleotide exchange factor 4 . 
A

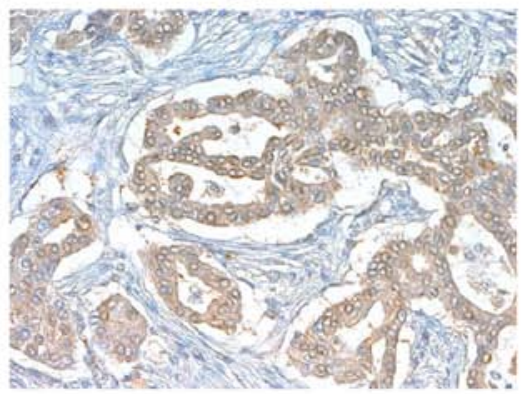

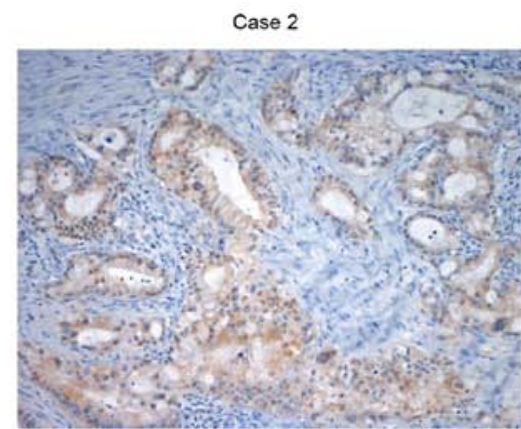

c

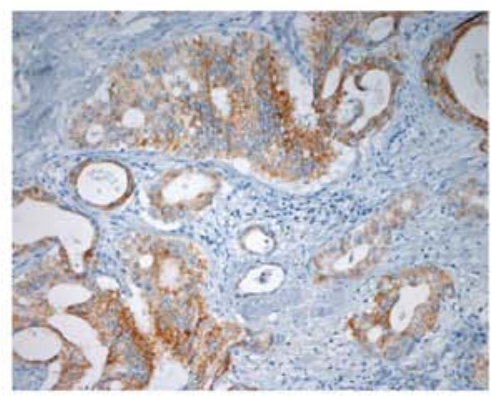

E

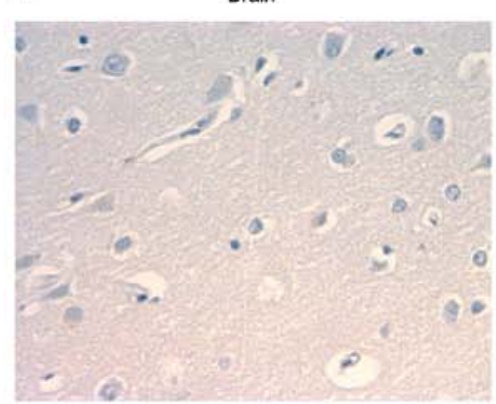

Liver

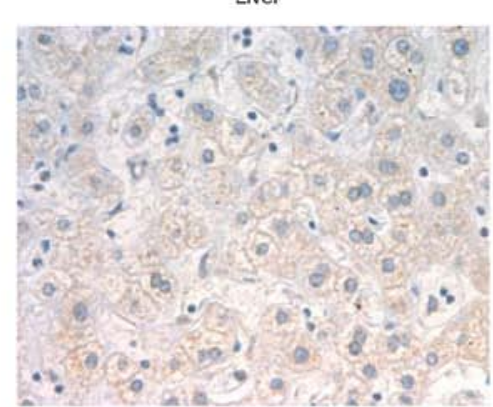

PanlN

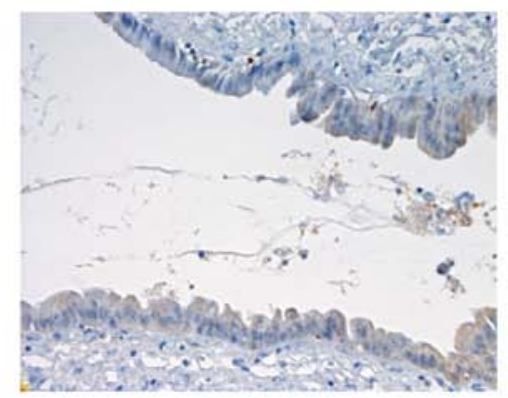

D

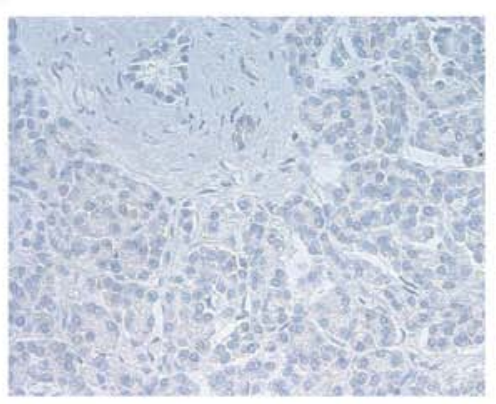

Figure 1. Immunohistochemistry with anti-ARHGEF4 antibody. (A) Immunohistochemical staining of two PDAC tissues using anti-ARHGEF4 antibody. (B) Immunohistochemical staining of a PanIN tissue using anti-ARHGEF4 antibody. (C) Immunohistochemical staining of ARHGEF4 at the invasive front and at the central region of PDAC tumor. (D) Expression of ARHGEF4 in normal pancreas. (E) Representative ARHGEF4 staining of normal brain, lung, liver and kidney. Original magnification, x200. ARHGEF4, rho guanine nucleotide exchange factor 4; PDAC, pancreatic ductal adenocarcinoma; PanIN, pancreatic intraepithelial neoplasm. insoluble fraction increased in response to culture on fibronectin (Fig. 4A), which indicates that ARHGEF4 translocated from the cytosol to the actin cytoskeleton following culture on fibronectin.

To examine the effects of alteration of actin cytoskeleton dynamics on the subcellular distribution of ARHGEF4, S2-013 and PANC-1 cells were treated with the actin depolymerising agent, cytochalasin D. Peripheral actin structures were reduced in the fibronectin-stimulated S2-013 and
PANC-1 cells exposed to $100 \mu \mathrm{M}$ cytochalasin D for $12 \mathrm{~h}$ than in the fibronectin-stimulated non-treated cells, and ARHGEF4 was present in the cytoplasm of cell bodies in the treated cells (Fig. 4B). In the non-treated S2-013 and PANC-1 cells grown on fibronectin, ARHGEF4 was accumulated in the cell protrusions (Fig. 4B).

Roles of ARHGEF4 in cell motility and invasion of PDAC cells. To determine whether ARHGEF4 was associated with the 
Table II. Correlation between ARHGEF4 expression and clinicopathological parameters.

\begin{tabular}{|c|c|c|c|c|c|}
\hline \multirow[b]{3}{*}{ Parameter } & \multicolumn{4}{|c|}{ ARHGEF4 expression } & \multirow[b]{3}{*}{ P-value } \\
\hline & \multicolumn{2}{|c|}{ Low } & \multicolumn{2}{|c|}{ High } & \\
\hline & $\%$ of total & Patients, $\mathrm{n}$ & $\%$ of total & Patients, $\mathrm{n}$ & \\
\hline Stage $\mathrm{e}^{\mathrm{a}}$ & & & & & 0.912 \\
\hline 0 & 3.2 & 2 & 0.0 & 0 & \\
\hline IA & 4.7 & 3 & 2.6 & 1 & \\
\hline IB & 9.3 & 6 & 5.3 & 2 & \\
\hline IIA & 28.1 & 18 & 36.9 & 14 & \\
\hline IIB & 48.4 & 31 & 50.0 & 19 & \\
\hline III & 1.6 & 1 & 2.6 & 1 & \\
\hline IV & 4.7 & 3 & 2.6 & 1 & \\
\hline Primary tumor ${ }^{\mathrm{a}}$ & & & & 0.595 & \\
\hline Tis & 3.2 & 2 & 0.0 & 0 & \\
\hline $\mathrm{T} 1$ & 7.7 & 5 & 2.6 & 1 & \\
\hline $\mathrm{T} 2$ & 12.5 & 8 & 18.4 & 7 & \\
\hline $\mathrm{T} 3$ & 75.0 & 48 & 76.4 & 29 & \\
\hline $\mathrm{T} 4$ & 1.6 & 1 & 2.6 & 1 & \\
\hline Regional lymph nodes ${ }^{a}$ & & & & & 1 \\
\hline NO & 45.3 & 29 & 47.4 & 18 & \\
\hline $\mathrm{N} 1$ & 54.7 & 35 & 52.6 & 20 & \\
\hline Distant metastasis $^{\mathrm{a}}$ & & & & & 1 \\
\hline M0 & 95.3 & 61 & 97.4 & 37 & \\
\hline M1 & 4.7 & 3 & 2.6 & 1 & \\
\hline Histology $\mathrm{y}^{\mathrm{b}}$ & & & & & 0.117 \\
\hline PanIN & 3.2 & 2 & 0.0 & 0 & \\
\hline Well & 34.3 & 22 & 23.6 & 9 & \\
\hline Moderate & 56.2 & 36 & 55.3 & 21 & \\
\hline Poor & 6.3 & 4 & 21.1 & 8 & \\
\hline Venous invasion ${ }^{\mathrm{b}}$ & & & & & 0.173 \\
\hline $\mathrm{v} 0+\mathrm{v} 1$ & 87.5 & 56 & 84.2 & 32 & \\
\hline $\mathrm{v} 2+\mathrm{v} 3$ & 12.5 & 8 & 15.8 & 6 & \\
\hline Lymphatic invasion $^{\mathrm{b}}$ & & & & & 0.636 \\
\hline ly $0+$ ly 1 & 73.4 & 47 & 78.9 & 30 & \\
\hline ly $2+1 y 3$ & 26.6 & 17 & 21.1 & 8 & \\
\hline
\end{tabular}

${ }^{a}$ Classified according to the classification of International Union against Cancer; ${ }^{b}$ classified according to the classification of pancreatic cancer of Japan Pancreas Society. Tis, intraepithelial carcinoma; PanIN, pancreatic intraepithelial neoplasia; ARHGEF4, rho guanine nucleotide exchange factor 4 .

motility and invasiveness of PDAC cells, ARHGEF4 expression in S2-013 and PANC-1 cells was transiently suppressed by $A R H G E F 4$-specific siRNA (Fig. 5A). Suppression of ARHGEF4 significantly inhibited cell motility (Fig. 5B) and invasion (Fig. 5C) in S2-013 and PANC-1 cells. An ARHGEF4-rescue construct was transiently transfected into scrambled control-siRNA and ARHGEF4-siRNA transfected S2-013 cells, and expression of myc-tagged ARHGEF4 was confirmed (Fig. 5D). The exogenous ARHGEF4 from the rescue construct was localized in the cytoplasm of cell bodies and in cell protrusions, similar to endogenous
ARHGEF4 (Fig. 5E). ARHGEF4-siRNA transfected S2-013 and PANC-1 cells expressing the rescue construct could rescue cell motility and invasiveness inhibited following ARHGEF4 silencing (Fig. 5F and G). These results indicated that ARHGEF4 promoted PDAC cell motility and invasion.

Roles of ARHGEF4 informing cell protrusions. Peripheral actin structures in membrane ruffles of scrambled control-siRNA and ARHGEF4-siRNA transfected S2-013 and PANC-1 cells cultured on fibronectin were then analyzed. Peripheral actin structures in cell protrusions were less abundant in 
A

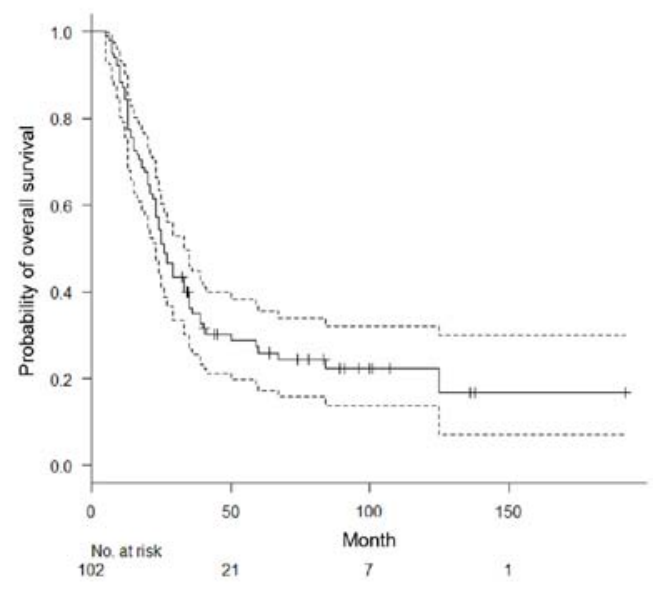

B
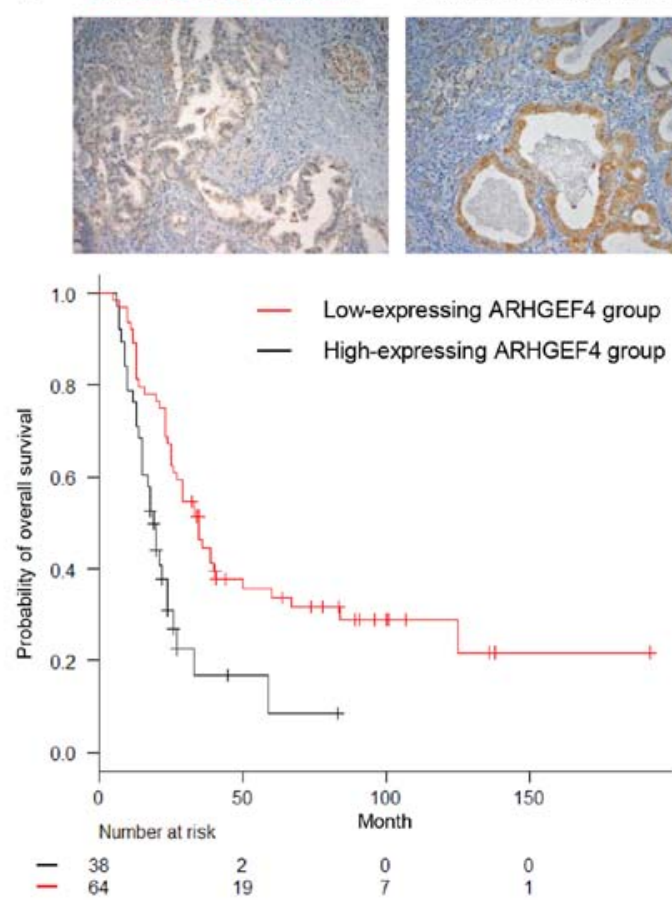

Figure 2. Correlation between high expression of ARHGEF4 and poor outcomes in PanIN and PDAC patients. (A) Kaplan-Meier analysis of overall survival of PDAC patients. The dashed lines are lower and upper limits of 95\% CI. (B) Kaplan-Meier analysis of overall survival of PDAC patients to ARHGEF4 expression and representative immunohistochemical staining of low-expressing ARHGEF4 and high-expressing ARHGEF4 PDAC tissues. Original magnification, x200. The median survival time was 26 months (95\% CI, 23-33). ARHGEF4, rho guanine nucleotide exchange factor 4; PDAC, pancreatic ductal adenocarcinoma; $\mathrm{CI}$, confidence interval.

A

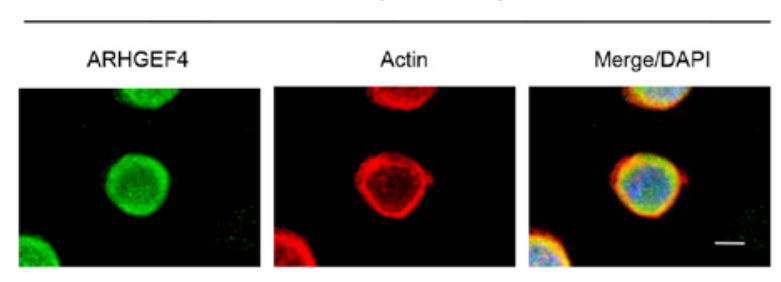

PANC-1 (fibronectin -)

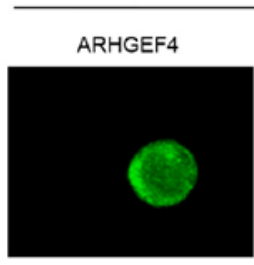

Actin

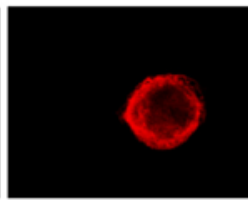

B

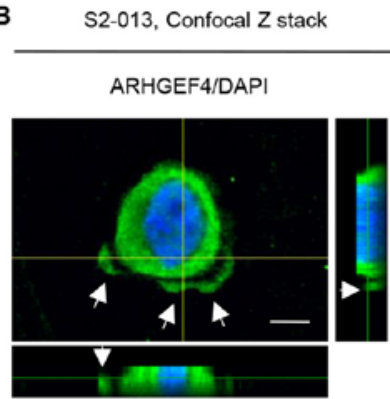

S2-013 (fibronectin +)

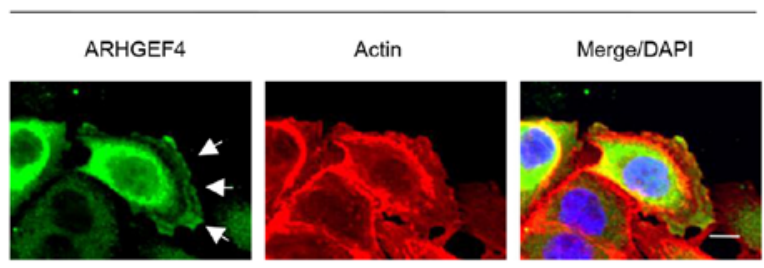

PANC-1 (fibronectin +)

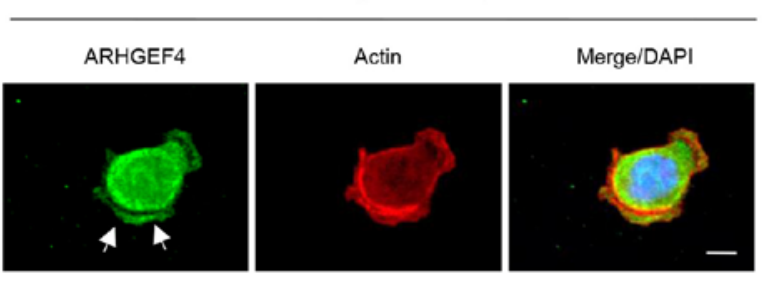

PANC-1, Confocal Z stack

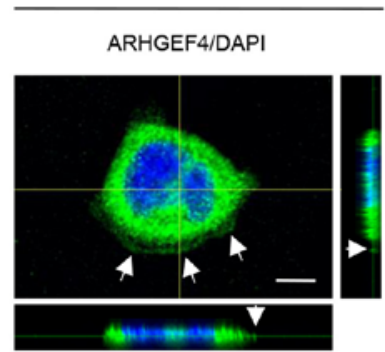

Figure 3. The distribution of ARHGEF4 in PDAC cells. (A) Immunocytochemistry on S2-013 and PANC-1 cells that were cultured with or without fibronectin. The cells were labeled with anti-ARHGEF4 antibody (green) and phalloidin (red; actin filaments). White arrows indicate ARHGEF4 localized in cell protrusions. Blue staining indicates DAPI staining. (B) Confocal Z stack showing nuclear DAPI staining (blue) and the accumulation of ARHGEF4 (green) in the membrane protrusions (white arrows) of S2-013 and PANC-1 cells grown on fibronectin. The lower and right panels in the confocal Z stack indicate a vertical cross-section (yellow lines) through the cells. Scale bars, $10 \mu \mathrm{m}$. ARHGEF4, rho guanine nucleotide exchange factor 4; PDAC, pancreatic ductal adenocarcinoma. 
Table III. Univariate and multivariate analysis of prognostic factors for overall survival.

\begin{tabular}{|c|c|c|c|c|}
\hline \multirow[b]{3}{*}{ Parameter } & \multicolumn{4}{|c|}{ Overall survival } \\
\hline & \multicolumn{2}{|c|}{ Univariate } & \multicolumn{2}{|c|}{ Multivariate } \\
\hline & $\mathrm{HR}(95 \% \mathrm{CI})$ & P-value & $\mathrm{HR}(95 \% \mathrm{CI})$ & P-value \\
\hline \multicolumn{5}{|l|}{ Stage $^{\mathrm{a}}$} \\
\hline $0+\mathrm{IA}+\mathrm{IB}$ & 1.0 (reference) & 0.549 & 1.0 (reference) & \\
\hline IIA & $1.159(0.714-1.881)$ & 0.196 & $4.638(1.593-13.50)$ & 0.004 \\
\hline IIB & $1.356(0.854-2.151)$ & 0.010 & $5.446(1.911-15.52)$ & 0.001 \\
\hline $\mathrm{III}+\mathrm{IV}$ & $3.035(1.301-7.081)$ & & $13.71(3.748-50.18)$ & $<0.001$ \\
\hline Age & $1.021(0.995-1.048)$ & 0.110 & $1.015(0.989-1.043)$ & 0.255 \\
\hline Sex & $1.107(0.696-1.761)$ & 0.666 & $1.192(0.742-1.913)$ & 0.468 \\
\hline ARHGEF4 expression & $0.345(0.213-0.559)$ & $<0.001$ & $0.411(0.239-0.706)$ & 0.001 \\
\hline Diameter of primary tumor & $1.338(1.176-1.524)$ & $<0.001$ & & \\
\hline Histology & $1.383(0.846-2.261)$ & 0.196 & & \\
\hline Lymphatic invasion $^{\mathrm{b}}(\mathrm{ly} 0+\mathrm{ly} 1$ or $1 \mathrm{y} 2+\mathrm{ly} 3)$ & $1.269(0.751-2.145)$ & 0.373 & & \\
\hline Venous invasion $^{\mathrm{b}}(\mathrm{v} 0+\mathrm{v} 1$ or v2+v3) & $1.928(1.034-3.593)$ & 0.038 & & \\
\hline Intrapancreatic nerve invasion $^{\mathrm{b}}(\mathrm{n} 0+\mathrm{n} 1$ or $\mathrm{n} 2+\mathrm{n} 3)$ & $1.500(0.947-2.377)$ & 0.083 & & \\
\hline
\end{tabular}

${ }^{a}$ Classified according to the classification of International Union against Cancer; ${ }^{b}$ classified according to the classification of pancreatic cancer of Japan Pancreas Society. HR, hazard ratio; CI, confidence interval.

A

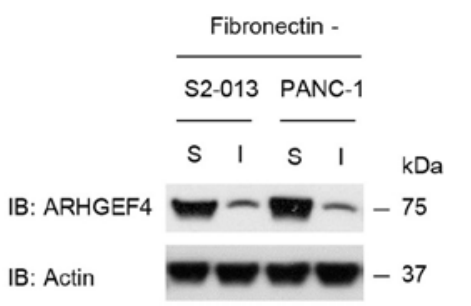

B

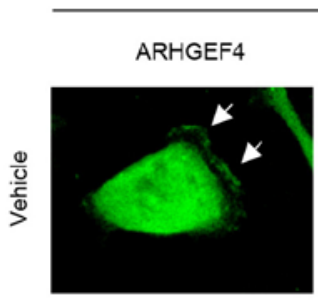

S2-013 cells grown on fibronectin

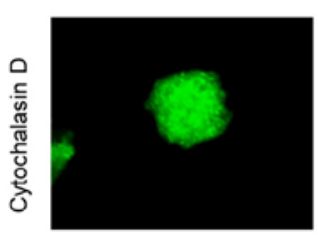

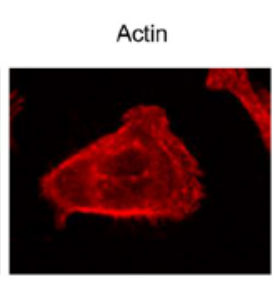

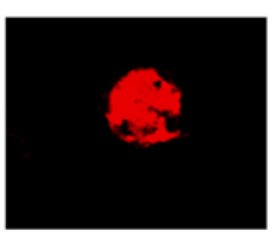

$1.159(0.714-1.881)$

$1.356(0.854-2.151)$

$1.021(0.995-1.048)$

$1.107(0.696-1.761)$

$1.383(0.846-2.261)$

0.196

$1.928(1.034-3.593)$ (1) 
A

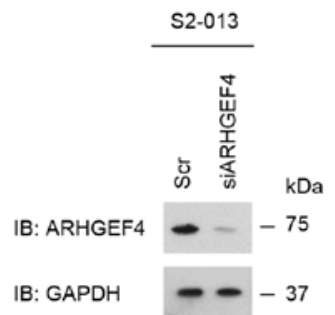

B

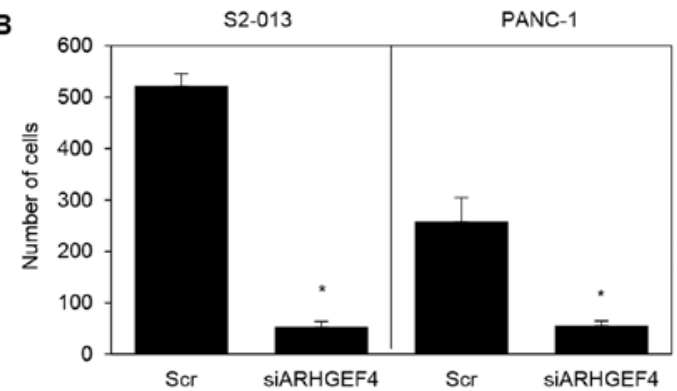

PANC-1

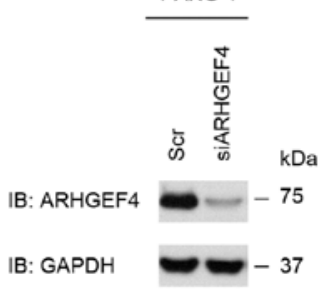

D

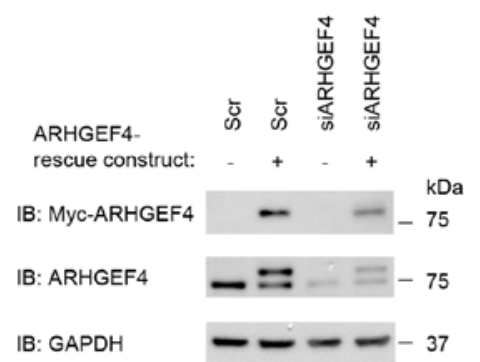

c

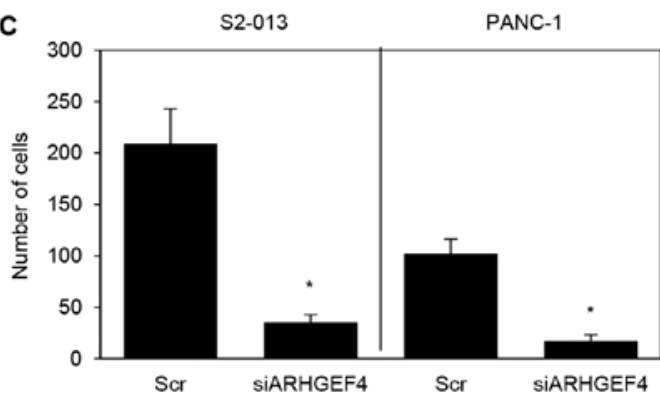

F

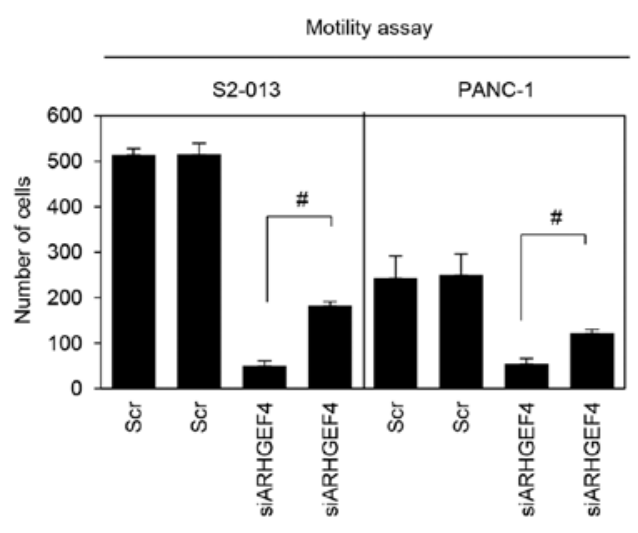

Transfection: Mock

Transfection: ARHGEF4

-rescue construct
E

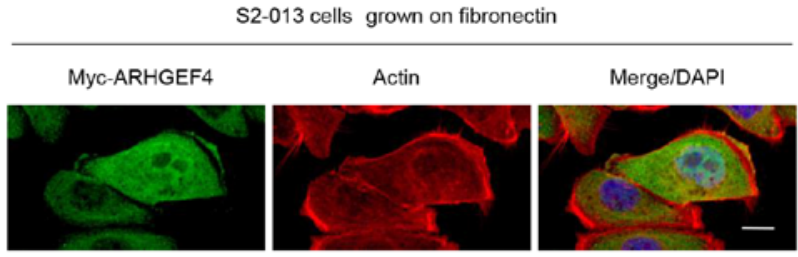

G
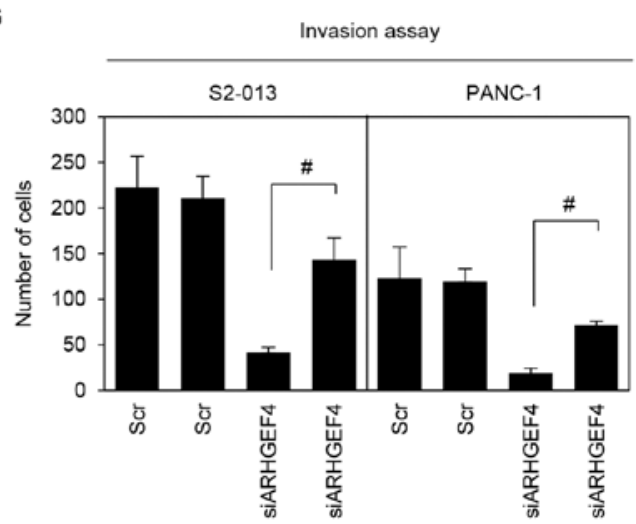

Figure 5. Role of ARHGEF4 in the motility and invasiveness of pancreatic ductal adenocarcinoma cells. (A) siARHGEF4 or Scr were transiently transfected into S2-013 and PANC-1 cells. Western blotting was performed using anti-ARHGEF4 antibodies. Scr or siARHGEF4 was transiently transfected into S2-013 and PANC-1 cells. (B) Motility and (C) two-chamber invasion assays were performed. Migrating cells in four fields per group were scored. Data were derived from three independent experiments. (D) Mock control vector or myc-tagged ARHGEF4-rescue construct were transiently transfected into Scr or siARHGEF4 transfected S2-013 cells. The myc-tagged ARHGEF4 was revealed by western blotting with an anti-myc antibody. (E) S2-013 cells were transfected with a myc-tagged ARHGEF4-rescue construct, incubated on fibronectin and analyzed by confocal immunofluorescence, using anti-myc antibody (green). Actin filaments were labeled by phalloidin (red). Blue staining indicates DAPI staining. Scale bar, $10 \mu \mathrm{M}$. Mock control vector or myc-tagged ARHGEF4-rescue constructs were transiently transfected into Scr or siARHGEF4 transfected S2-013 and PANC-1 cells, and $48 \mathrm{~h}$ later (F) motility and (G) two-chamber invasion assays were performed. Migrating cells in four fields per group were counted. Data were derived from three independent experiments. Data are presented as the mean \pm standard deviation. ${ }^{*} \mathrm{P}<0.005$ vs. Scr-transfected control; ${ }^{\#} \mathrm{P}<0.01$. ARHGEF4, rho guanine nucleotide exchange factor 4 ; siRNA, small interfering RNA; siARHGEF4, siRNA oligonucleotides targeting ARHGEF4; Scr, negative control scrambled siRNAs.

transfected S2-013 and PANC-1 cells rescued the decrease in peripheral actin structures at the protrusions caused by the ARHGEF4-siRNA (S2-013, Fig. 6C; PANC-1, Fig. 6D).
To quantify the degree of inhibition caused by suppression of ARHGEF4, the induction of membrane protrusions was observed. In ARHGEF4-siRNA transfected S2-013 
A

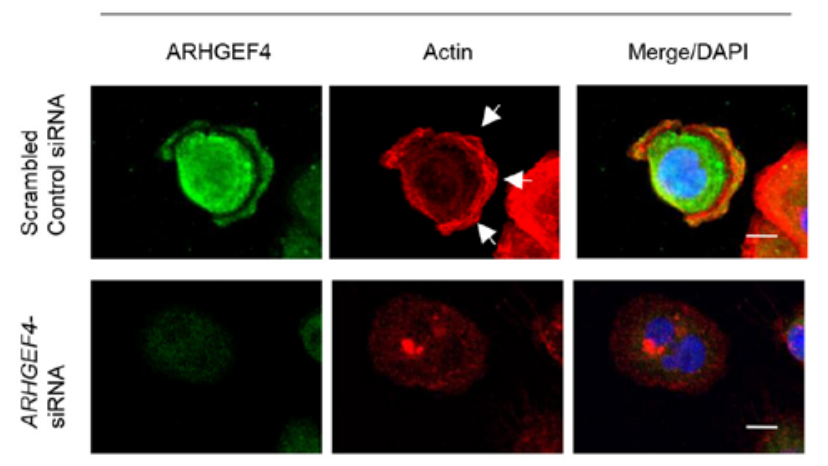

C

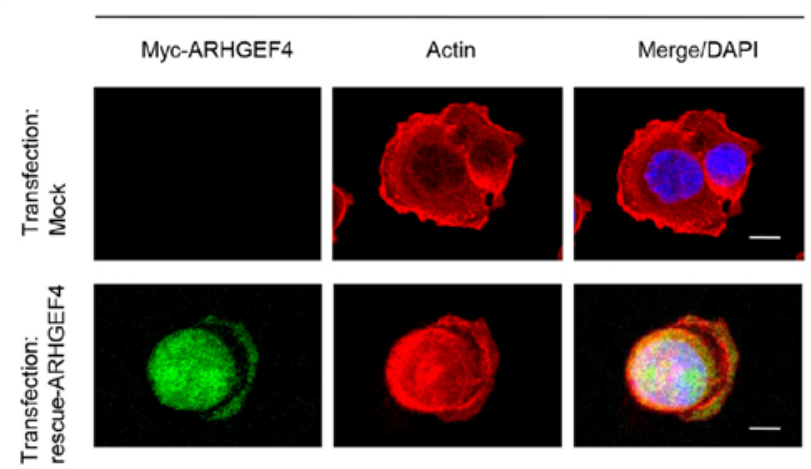

D

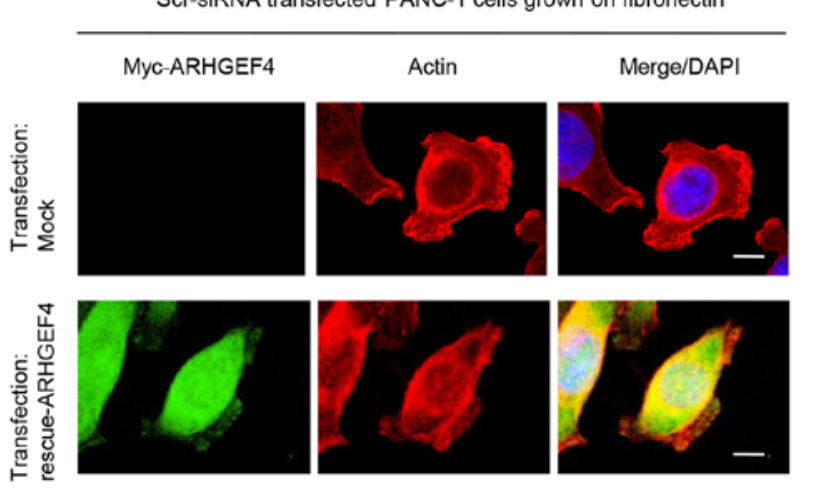

B

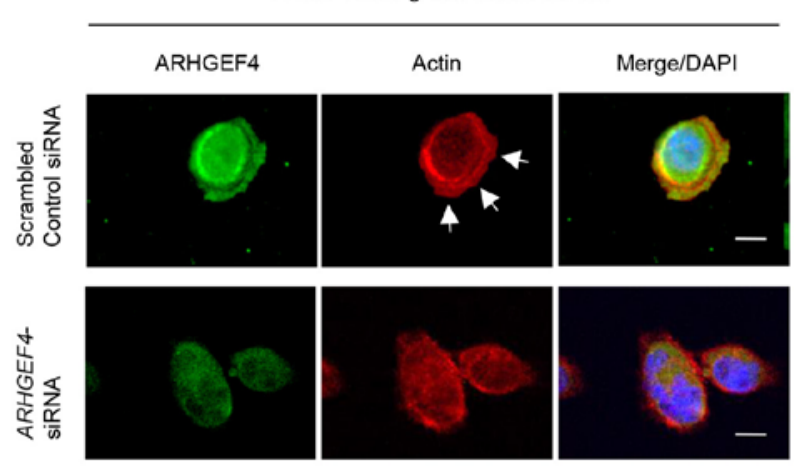

ARHGEF4-siRNA transfected S2-013 cells grown on fibronectin

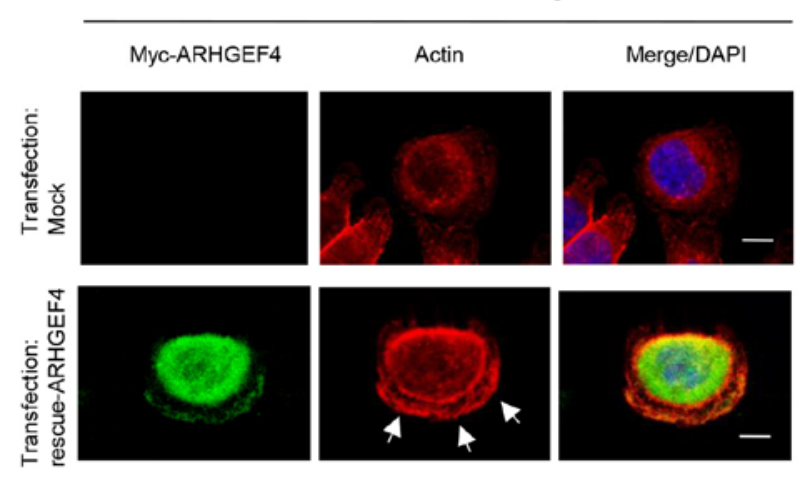

ARHGEF4-siRNA transfected PANC-1 cells grown on fibronectin

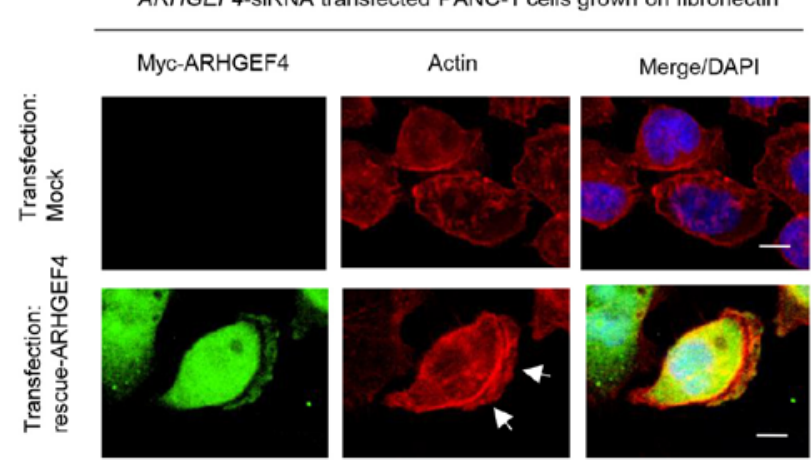

Figure 6. Roles of ARHGEF4 in the peripheral actin rearrangements. Confocal immunofluorescence microscopic images. siARHGEF4 or Scr were transiently transfected into (A) S2-013 and (B) PANC-1 cells. The cells were incubated on fibronectin and screened with anti-ARHGEF4 antibody (green) and phalloidin (red). Arrows indicate peripheral actin structures in cell protrusions of Scr-transfected cells. The mock control vector or myc-tagged ARHGEF4-rescue construct were transiently transfected into siARHGEF4 or Scr transfected (C) S2-013 and (D) PANC-1 cells. Following 48 h, the cells were incubated on fibronectin. Cells were stained with anti-myc antibody (green). Actin filaments were labeled by phalloidin (red). Arrows indicate cell protrusions reinstated by exogenous ARHGEF4 in ARHGEF4-siRNA transfected cells. Blue staining indicates DAPI staining. Scale bars, $10 \mu \mathrm{M}$. ARHGEF4, rho guanine nucleotide exchange factor 4; siRNA, small interfering RNA; siARHGEF4, siRNA oligonucleotides targeting ARHGEF4; Scr, negative control scrambled siRNAs.

and PANC-1 cells cultured on fibronectin, suppression of ARHGEF4 inhibited the formation of protrusions compared with control-siRNA transfected S2-013 and PANC-1 cells (S2-013, Fig. 7A; PANC-1, Fig. 7B). Transfection of an ARHGEF4-rescue construct into ARHGEF4-siRNA transfected S2-013 and PANC-1 cells rescued the decrease in the protrusions caused by the ARHGEF4-siRNA (Fig. 7). These results indicated that $\mathrm{ARHGEF} 4$ regulates the rearrangement of peripheral actin to induce the formation of additional membrane protrusions.

Links of ARHGEF4 with associated cell signaling pathways. To determine whether ARHGEF4 could regulate the activity of phosphoproteins, the intracellular signaling pathways were further analyzed in the scrambled control-siRNA transfected S2-013 cells and ARHGEF4-siRNA transfected S2-013 cells grown on fibronectin (Fig. 8). Of the 38 kinases investigated in a commercially available human phosphoprotein array kit, suppression of ARHGEF4 markedly downregulated the activity of Src, ERK1/2, p38 $\alpha$ and GSK-3 $\alpha / \beta$.

Association of ARHGEF4 with ERK1/2 in the formation of cell protrusions. To evaluate the role of the ERK1/2 pathway on the ARHGEF4-mediated formation of cell protrusions, the actin cytoskeletal structures of the scrambled controlsiRNA transfected S2-013 and PANC-1 cells as well as the 

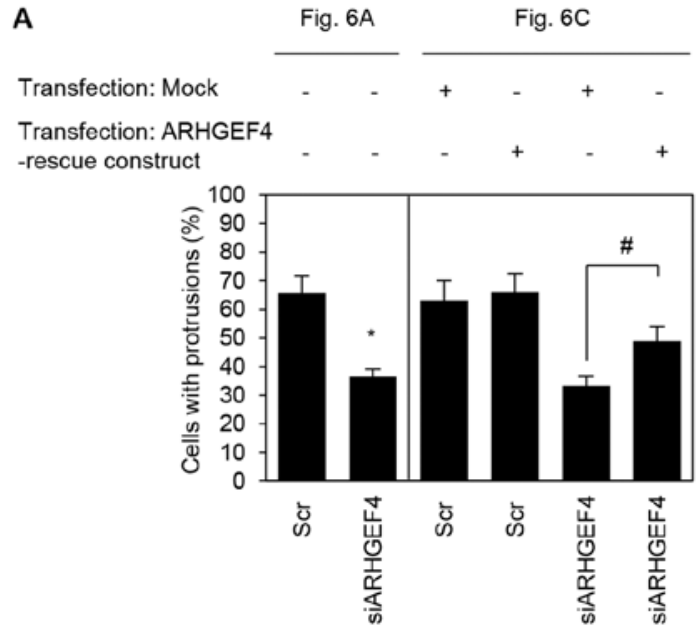

B
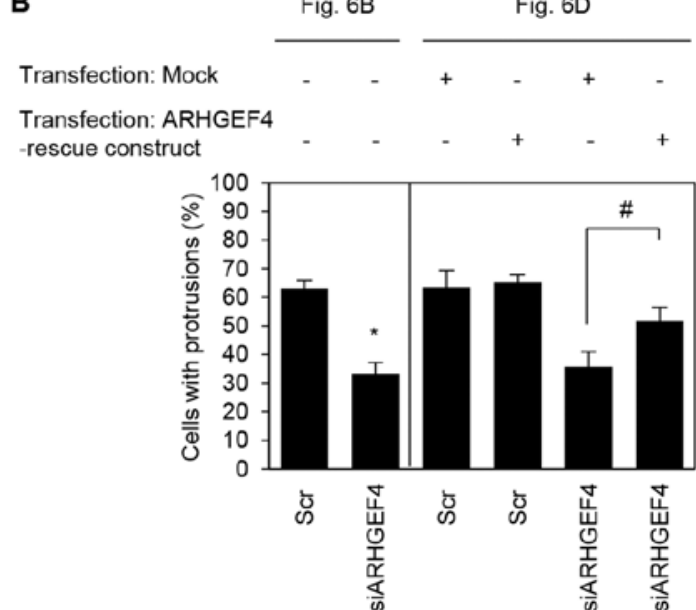

Figure 7. Quantification of the frequency of formation of actin-rich protrusions. Quantification of the data presented in (A) Fig. 6A and C, and (B) Fig. 6B and D. The values represent the number of cells with fibronectin-stimulated cell protrusions in which peripheral actin structures were increased. All cells in four fields per group were scored. Data were derived from three independent experiments. Data are presented as the mean \pm standard deviation. " $\mathrm{P}<0.01$ vs. Scr-transfected control; " $\mathrm{P}<0.05$. ARHGEF4, rho guanine nucleotide exchange factor 4; siRNA, small interfering RNA; siARHGEF4, siRNA oligonucleotides targeting ARHGEF4; Scr, negative control scrambled siRNAs.

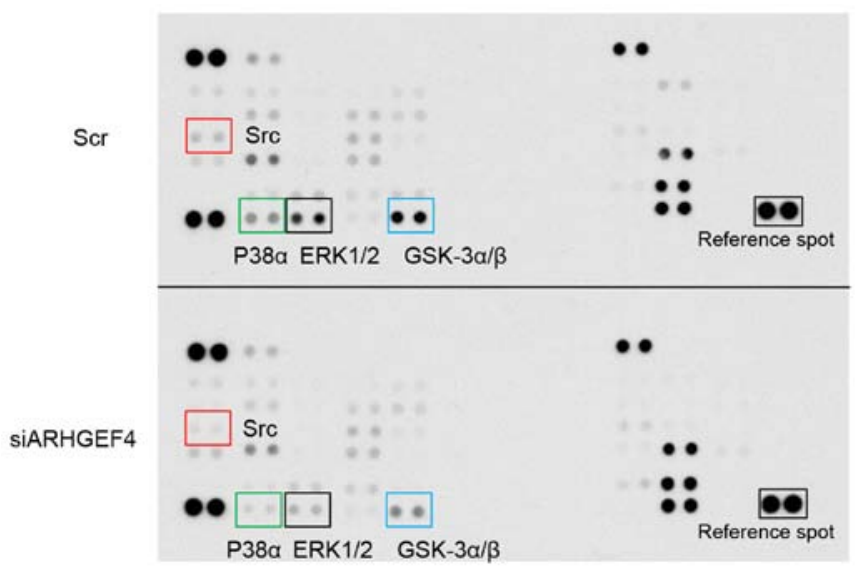

Figure 8. Analysis of cell signaling pathways that may be associated with ARHGEF4 activities. Human phosphoprotein arrays were used to examine the activity of phosphoproteins levels in lysates from Scr transfected S2-013 cells grown on fibronectin as a control and from siARHGEF4 transfected S2-013 cells grown on fibronectin. Data are representative of three independent experiments. ARHGEF4, rho guanine nucleotide exchange factor 4; siRNA, small interfering RNA; siARHGEF4, siRNA oligonucleotides targeting ARHGEF4; Scr, negative control scrambled siRNAs; ERK, extracellular signal-regulated kinase; GSK, glycogen synthase kinase.

ARHGEF4-siRNA transfected S2-013 and PANC-1 cells grown on fibronectin in the absence or presence of the MEK inhibitor, U0126 were examined. Inhibition of MEK activity by treatment with U0126 has previously been reported to decrease downstream kinase target ERK1/2 phosphorylation, confirming the inhibition of the MEK signaling pathway (25). Treatment of S2-013 cells grown on fibronectin with U0126 inhibited ERK1/2 activity (Fig. 9A). The suppression of ARHGEF4 partially decreased ERK1/2 activity, and similarly, ERK1/2 phosphorylation was inhibited by U0126 at the concentration of $30 \mu \mathrm{M}$ (Fig. 9A). Immunocytochemistry demonstrated that treatment with U0126 decreased phosphorylated ERK1/2 localized to the cytoplasm of the cell bodies, and inhibited peripheral actin rearrangement in S2-013 cells grown on fibronectin (Fig. 9B). Notably, phosphorylated ERK1/2 was also present at cell protrusions of non-treated S2-013 cells (Fig. 9B). Additionally, suppression of ARHGEF4 decreased phosphorylated ERK1/2 localized to the cytoplasm of the cell bodies and peripheral actin filaments in the cell protrusions when compared with those in S2-013 cells transfected with scrambled control-siRNA (Fig. 9C). Transfection of an ARHGEF4-rescue construct into S2-013 and PANC-1 cells in which ARHGEF4 had been suppressed and ERK1/2 phosphorylation had been blocked by U0126 did not result in a marked increase in peripheral actin filaments in the cell protrusions when compared with those in cells transfected with the mock control vector (S2-013, Fig. 9D; PANC-1, Fig. 9E). These data indicated that the ERK pathway was largely responsible for the ARHGEF4-mediated formation of membrane ruffles in PDAC cells.

Association of ARHGEF4 with $G S K-3 \alpha / \beta$ in the formation of cell protrusions. The association of ARHGEF4 and GSK- $3 \alpha / \beta$ with the formation of membrane ruffles was evaluated. GSK-3 activity was downregulated using the GSK-3 inhibitor SB216763. GSK suppression increases the expression levels of cleaved caspase-8, cleaved caspase-3, and cleaved poly(ADP-ribose) polymerase $(26,27)$. SB216763 was potent in markedly reducing GSK-3 $\alpha / \beta$ activity, as visualized by an increased level of cleaved caspase- 8 in S2-013 cells grown on fibronectin (Fig. 10A). Cleaved caspase- 8 was also increased by the suppression of ARHGEF4 in S2-013 cells, compared with scrambled control-siRNA transfected cells (Fig. 10A). Immunocytochemistry demonstrated that treatment with SB216763 increased caspase-8 in the cytoplasm, and inhibited peripheral actin rearrangement in S2-013 cells grown on fibronectin (Fig. 10B), similar to ARHGEF4-siRNA transfected S2-013 cells (Fig. 10C). Transfection of an ARHGEF4-rescue construct into S2-013 and PANC-1 cells in which ARHGEF4 had been suppressed and GSK-3 $\alpha / \beta$ phosphorylation had been blocked by SB216763 did not result in an increase in peripheral actin filaments when compared with those in cells transfected 


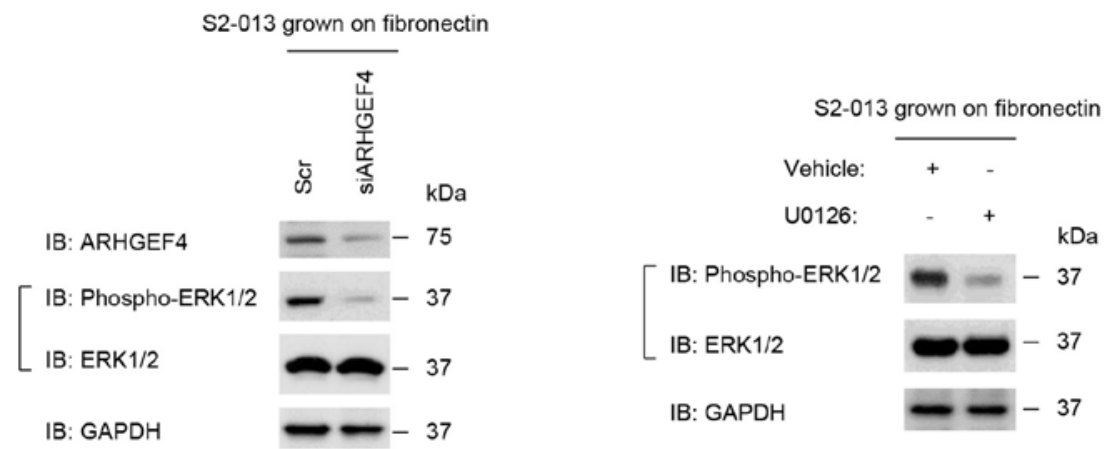

B
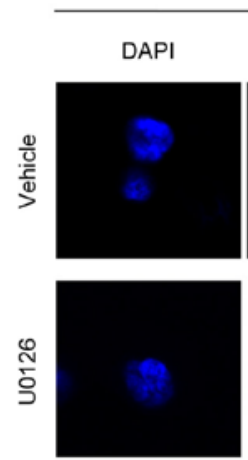

S2-013 cells grown on fibronectin
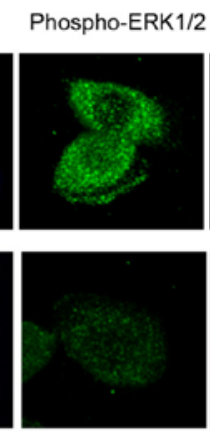
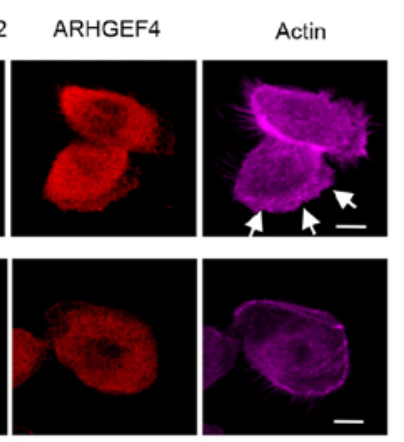

C

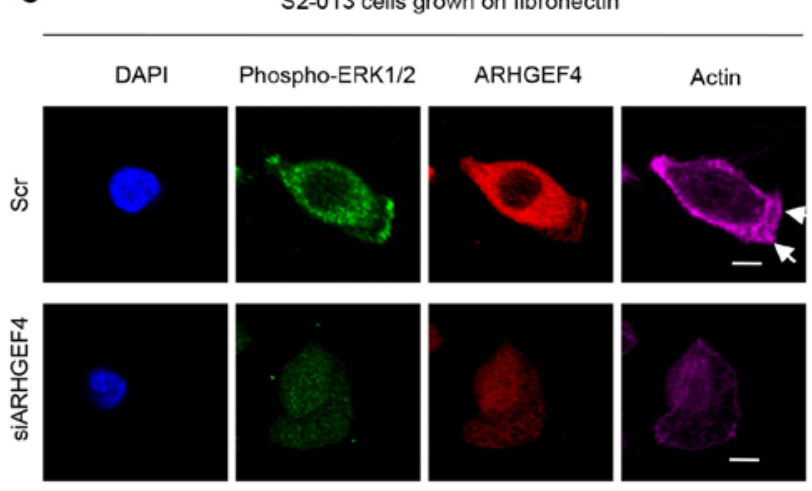

D

ARHGEF4-siRNA transfected S2-013 cells grown on fibronectin

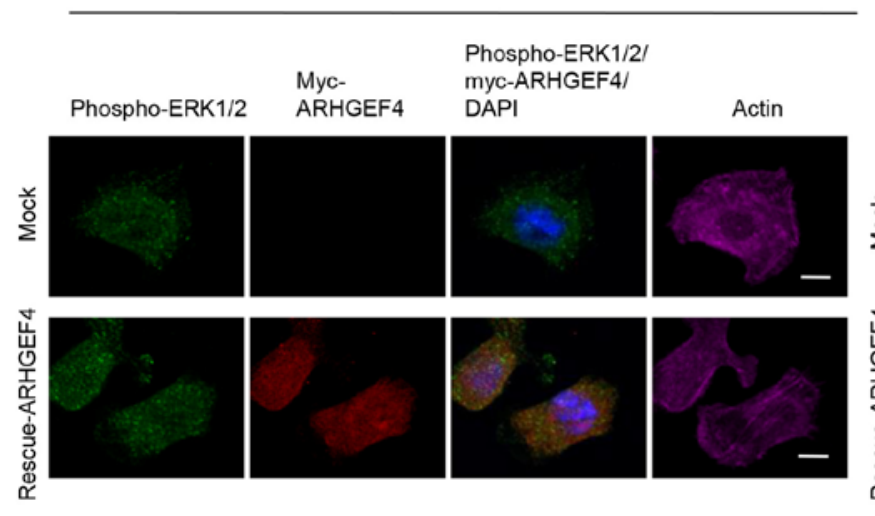

E ARHGEF4-siRNA transfected PANC-1 cells grown on fibronectin

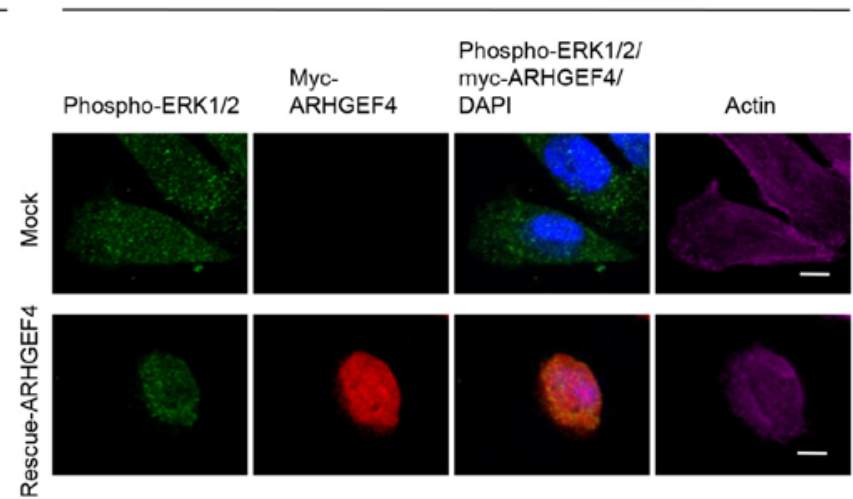

Figure 9. Association of ARHGEF4 with ERK1/2 in peripheral actin rearrangements. (A) S2-013 cells were incubated on fibronectin with or without U0126 treatment. Additionally, Scr transfected S2-013 cells and siARHGEF4 transfected S2-013 cells were grown on fibronectin. Western blotting of steadystate levels was performed using antibodies against ARHGEF4, ERK1/2 and phospho-ERK1/2. Data are representative of three independent experiments. (B) Confocal immunofluorescence microscopic images. S2-013 cells were incubated on fibronectin with or without U0126 treatment. The cells were immunocytochemically stained with anti-phospho-ERK1/2 antibody (green), anti-ARHGEF4 antibody (red) and phalloidin (violet). (C) Confocal immunofluorescence microscopic images. Scr transfected S2-013 cells and siARHGEF4 transfected S2-013 cells incubated on fibronectin were immunocytochemically stained with anti-phospho-ERK1/2 antibody (green), anti-ARHGEF4 antibody (red) and phalloidin (violet). Confocal immunofluorescence microscopic images. A myc-tagged ARHGEF4-rescue construct was transfected into (D) S2-013 and (E) PANC-1 cells that had been transfected with siARHGEF4; 48 h later, the cells were incubated on fibronectin with or without U0126 treatment. Cells were stained with anti-phospho-ERK1/2 antibody (green), anti-myc antibody (red) and phalloidin (violet). Blue staining indicates DAPI staining. Arrows indicate cell protrusions in which the peripheral actin structures are increased. Scale bars, $10 \mu \mathrm{M}$. ARHGEF4, rho guanine nucleotide exchange factor 4; ERK, extracellular signal-regulated kinase; phospho-, phosphorylated; siRNA, small interfering RNA; Scr, negative control scrambled siRNAs; siARHGEF4, siRNA oligonucleotides targeting ARHGEF4.

with the mock-control vector (S2-013, Fig. 10D; PANC-1, Fig. 10E). These results indicated that ARHGEF4 serves a role in promoting peripheral actin cytoskeletal rearrangements by increasing the levels of phosphorylated GSK-3 $\alpha / \beta$.

Association of ARHGEF4 with ERK1/2 and GSK-3 $\alpha / \beta$ in the cell motility and invasion of PDAC cells. To evaluate whether ERK1/2 is necessary for ARHGEF4 to promote cell motility and invasion, motility and two-chamber invasion assays were performed using S2-013 and PANC-1 cells with or without U0126 pretreatment. S2-013 and PANC-1 cells pretreated with U0126 demonstrated significantly decreased cell motility and invasion when compared with the corresponding nontreated cells (Fig. 11A). Transfection of an ARHGEF4-rescue construct into S2-013 and PANC-1 cells in which ARHGEF4 had been suppressed and ERK1/2 had been inactivated did 


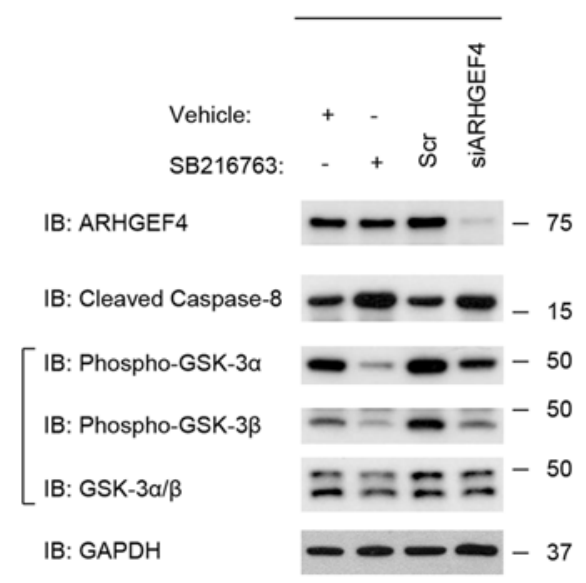

C

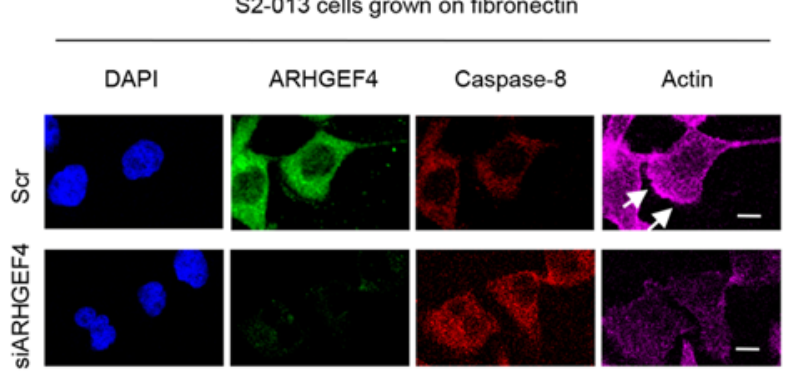

E ARHGEF4-siRNA transfected PANC-1 cells grown on fibronectin

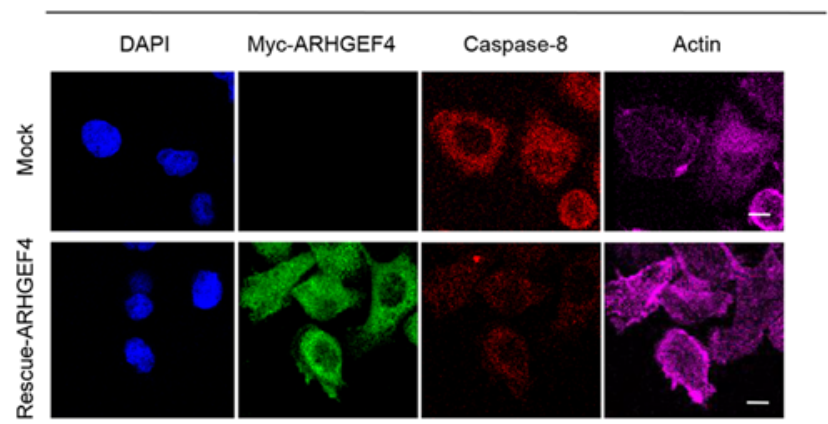

B

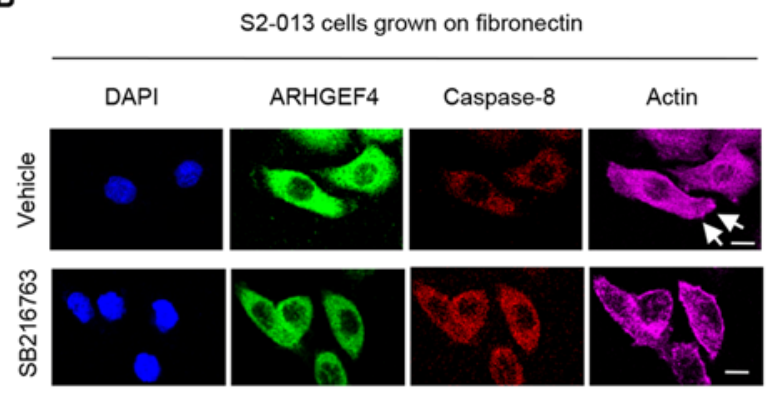

D

ARHGEF4-siRNA transfected S2-013 cells grown on fibronectin

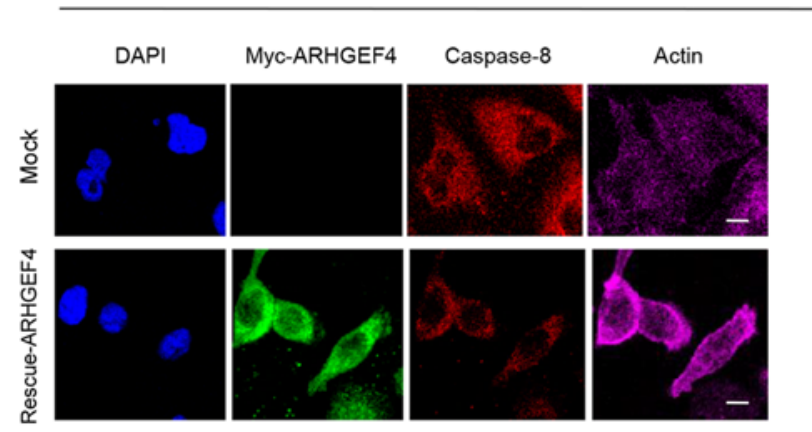

Figure 10. Association of ARHGEF4 with GSK-3 $\alpha / \beta$ in forming cell protrusions. (A) S2-013 cells were incubated on fibronectin with or without SB216763 treatment. Additionally, Scr transfected S2-013 cells and siARHGEF4 transfected S2-013 cells were grown on fibronectin. Western blotting of steadystate levels was performed using anti-ARHGEF4, anti-GSK-3 $\alpha / \beta$ and anti-caspase- 8 antibodies. Data are representative of three independent experiments. (B) Confocal immunofluorescence microscopic images. S2-013 cells were incubated on fibronectin with or without SB216763 treatment. The cells were immunocytochemically stained with anti-ARHGEF4 antibody (green), anti-caspase-8 antibody (red) and phalloidin (violet). (C) Confocal immunofluorescence microscopic images. Scr transfected S2-013 cells and siARHGEF4 transfected S2-013 cells incubated on fibronectin were immunocytochemically stained with anti-ARHGEF4 antibody (green), anti-caspase-8 antibody (red) and phalloidin (violet). Confocal immunofluorescence microscopic images. A myc-tagged ARHGEF4-rescue construct was transfected into (D) S2-013 and (E) PANC-1 cells that had been transfected with siARHGEF4; $48 \mathrm{~h}$ later, the cells were incubated on fibronectin with or without SB216763 treatment. Cells were stained with anti-myc antibody (green), anti-caspase-8 antibody (red) and phalloidin (violet). Blue staining indicates DAPI staining. Arrows indicate cell protrusions in which the peripheral actin structures are increased. Scale bars, $10 \mu \mathrm{M}$. ARHGEF4, rho guanine nucleotide exchange factor 4; GSK, glycogen synthase kinase; siRNA, small interfering RNA; Scr, negative control scrambled siRNAs; siARHGEF4, siRNA oligonucleotides targeting ARHGEF4.

not significantly abrogate the changes to cell motility and invasiveness caused by the suppression of ARHGEF4 and inactivation of ERK1/2 (S2-013, Fig. 11B; PANC-1, Fig. 11C). These results indicated that ERK1/2 was necessary for the ARHGEF4-associated promotion of motility and invasiveness.

To evaluate the association of ARHGEF4 and GSK- $3 \alpha / \beta$ with motility and invasiveness, motility and two-chamber invasion assays were performed using S2-013 and PANC-1 cells with or without SB216763 pretreatment. S2-013 and PANC-1 cells pretreated with U0126 demonstrated significantly decreased cell motility and invasion when compared with the corresponding non-treated cells (Fig. 12A). Transfection of an ARHGEF4-rescue construct into S2-013 and PANC-1 cells in which ARHGEF4 had been suppressed and GSK-3 $\alpha / \beta$ had been inactivated did not significantly abrogate the changes to cell motility and invasiveness caused by the suppression of 
A
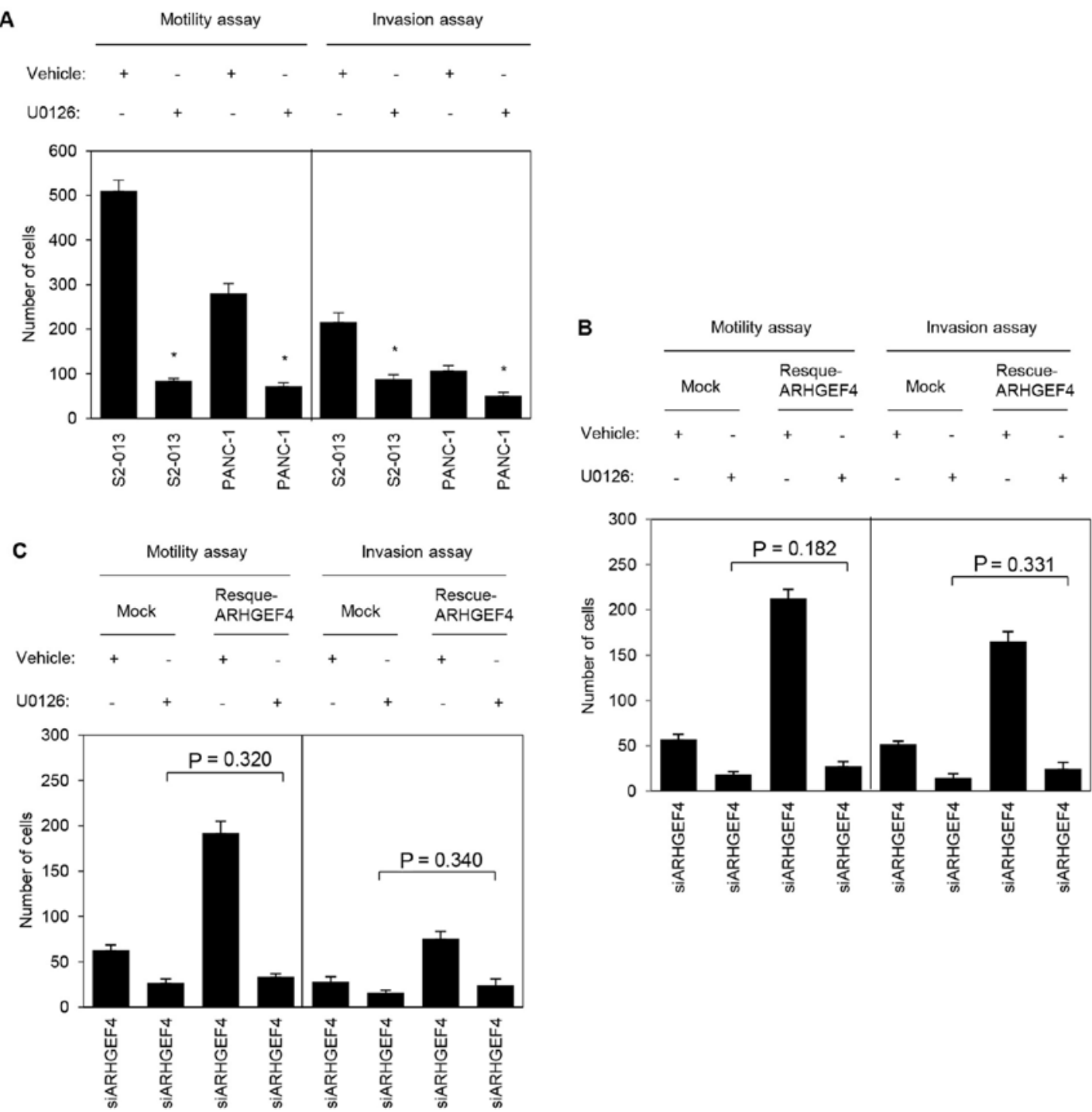

Figure 11. Association of ARHGEF4 with ERK1/2 in cell motility and invasion. (A) S2-013 and PANC-1 cells were pretreated with or without U0126 and then subjected to motility and two-chamber invasion assays. Migrating cells in four fields per group were scored. Data are representative of three independent experiments. A myc-tagged ARHGEF4-rescue construct was transfected into (B) S2-013 and (C) PANC-1 cells that had been transfected with siARHGEF4; $48 \mathrm{~h}$ later, the cells were treated with or without U0126. Motility and two-chamber invasion assays were performed. Migrating cells in four fields per group were scored. Data are representative of three independent experiments. Data are presented as the mean \pm standard deviation. "P $<0.05$ vs. non-treated cells. ARHGEF4, rho guanine nucleotide exchange factor 4; ERK, extracellular signal-regulated kinase; siRNA, small interfering RNA; siARHGEF4, siRNA oligonucleotides targeting ARHGEF4.

ARHGEF4 and inactivation of GSK-3 $\alpha / \beta$ (S2-013, Fig. 12B; PANC-1, Fig. 12C). These results indicated that GSK-3 $\alpha / \beta$ was necessary for the ARHGEF4-associated promotion of motility and invasiveness.

\section{Discussion}

In the present study, it was demonstrated that high ARHGEF4 expression is closely associated with poor prognosis of patients with PDAC. PDAC is one of the deadliest of all solid malignancies, with a 5-year survival rate of $8 \%$, due to its ability to extensively invade surrounding tissues and to metastasize at an early stage $(28,29)$. Multivariate Cox regression analysis indicated that high ARHGEF4 expression was an independent predictor of worse overall survival in a model including other clinicopathological factors. Table II demonstrated that there was no statistically significant relationship between
ARHGEF4 expression and any of the other clinicopathological factors; this may be attributable to the short overall survival rate of the high-expressing ARHGEF4 group, in which the median survival time and 3-year survival rate were 24 months and $26.3 \%$, respectively. In vitro motility and invasion assays demonstrated that ARHGEF4 promoted cell motility and invasion through an increase in cell protrusions in PDAC cells. These results indicate that ARHGEF4 may be a useful marker of poor prognosis of PDAC patients that is functionally associated with cell motility and invasion.

ARHGEF4 is a GEF for Rac1 and a link between APC and G-protein signaling $(1,3)$. ARHGEF4 transduces a Wnt signal to Rac1, thereby regulating the actin cytoskeleton and cell migration (4). The crystal structure of ARHGEF4 has been determined and it was demonstrated that the $\mathrm{SH} 3$ domain in the $\mathrm{NH}_{2}$-terminal regulatory domain serves a crucial role in the auto-inhibition of GEF activity (3). Epidermal growth 
A
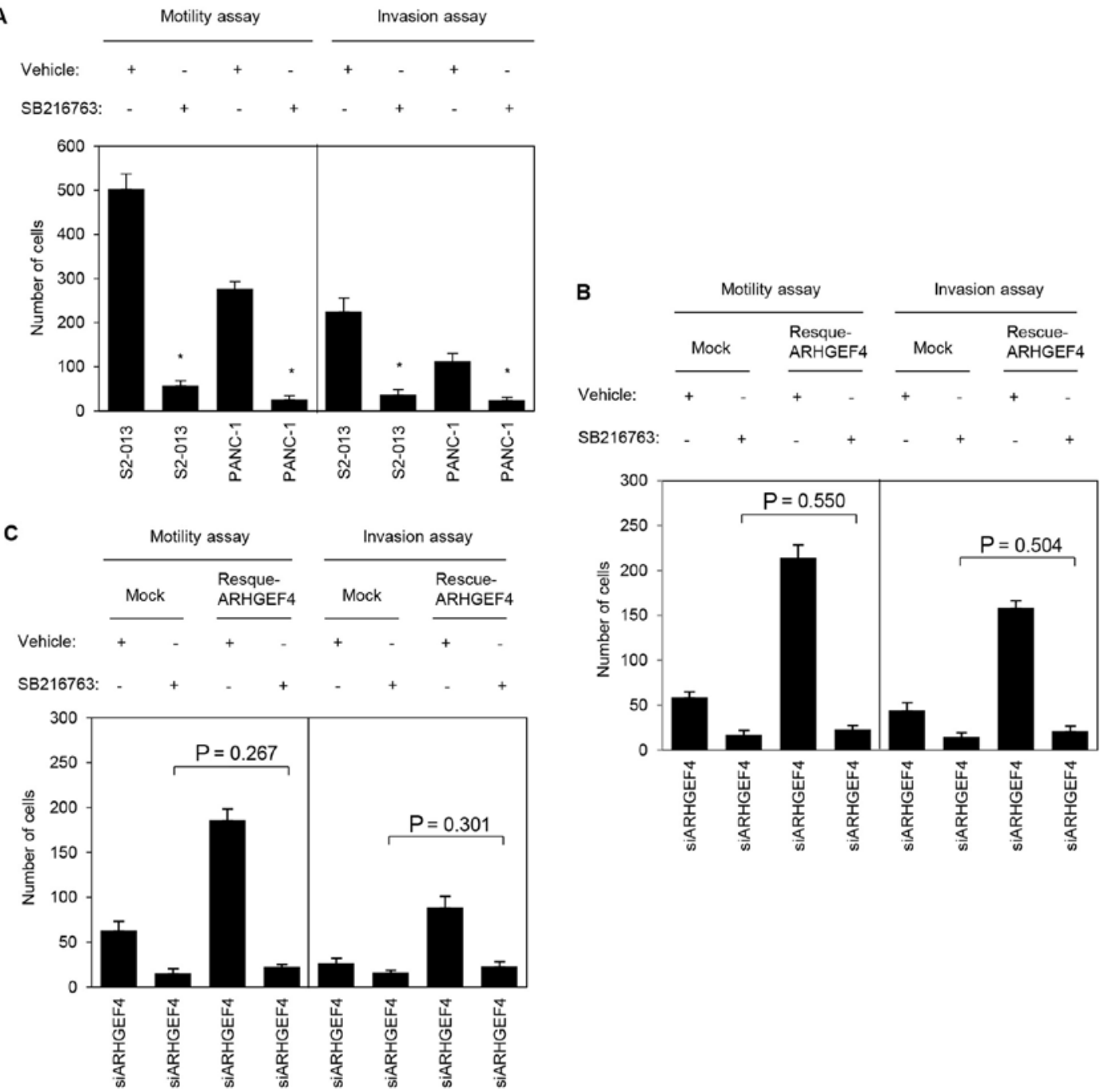

Figure 12. Association of ARHGEF4 with GSK-3 $\alpha / \beta$ in cell motility and invasion. (A) S2-013 and PANC-1 cells were pretreated with or without SB216763 and then subjected to motility and two-chamber invasion assays. Migrating cells in four fields per group were scored. A myc-tagged ARHGEF4-rescue construct was transfected into (B) S2-013 and (C) PANC-1 cells that had been transfected with siARHGEF4; $48 \mathrm{~h}$ later, the cells were treated with or without SB216763. Motility and two-chamber invasion assays were performed. Migrating cells in four fields per group were scored. Data are representative of three independent experiments. Data are presented as the mean \pm standard deviation. "P<0.05 vs. non-treated cells. ARHGEF4, rho guanine nucleotide exchange factor 4; GSK, glycogen synthase kinase; siRNA, small interfering RNA; siARHGEF4, siRNA oligonucleotides targeting ARHGEF4.

factor stimulation phosphorylates ARHGEF4 at Tyr94 within the APC-binding region in a Src kinase-dependent manner; however, no increase in GEF activity upon ARHGEF4 tyrosine phosphorylation or upon the introduction of a phospho-mimetic amino acid into Tyr94 of ARHGEF4 has been observed (30). This finding suggested that ARHGEF4 may serve different roles besides being a GEF, and it was demonstrated that ARHGEF4 knockdown inactivated Src, ERK1/2, p38 $\alpha$ and GSK-3 $\alpha / \beta$ in S2-013 cells. As such, in the present study, the investigation and discussion was limited to only how ARHGEF4 promotes the motility and invasiveness through ERK1/2 and GSK-3 $\alpha / \beta$, and the GEF activity of ARHGEF4 was not subjected to further investigation.

Src is found at the crossroads of several signaling pathways, including Ras/Raf/ERK1/2 (31-33), and it promotes cell proliferation, survival, invasion, migration and angiogenesis. The p38 and GSK- $3 \alpha / \beta$ pathways promote the proliferation, migration, and invasion of lung cancer cells (34). In PDAC cells, an antioxidant defense enzyme, Prdx1, is associated with the formation of membrane protrusions through the modulation of p38-MAPK activity, which in turn promotes cell invasion (35). Inhibition of GSK-3 impairs PDAC growth (36), thus providing a rationale for assessing the potential clinical utility of GSK3 inhibitors in PDAC patients (37). These findings indicated that ARHGEF4-associated actin remodeling and cell motility and invasiveness may be modulated through the regulation of multiple pathways, including Src, MAPK-ERK1/2, p38-MAPK and WNT-GSK, in PDAC cells. Future studies should evaluate the ARHGEF4-associated Src, MAPK-ERK1/2, p38-MAPK, and WNT-GSK pathways that coordinate the actin cytoskeletal remodeling that is required for cell spreading, motility, and invasion.

In conclusion, ARHGEF4 promotes cell motility and invasion, and may be a useful marker for predicting the outcome of patients with PDAC. Mechanistically, the present study provides the first evidence, to the best of our knowledge, that ARHGEF4-mediated phosphorylation of ERK1/2 and GSK-3 $\alpha / \beta$ may regulate the formation of cell protrusions, 
resulting in the promotion of PDAC cell motility and invasion. In addition, the data presented herein indicated that the i) inhibition of ARHGEF4, ii) inhibition of ERK1/2 and/or GSK-3 $\alpha / \beta$ pathways that associate with ARHGEF4, or iii) some combination thereof may be effective for development of new therapeutic strategies, as any such therapy would inhibit the ARHGEF4-mediated cell motility and invasion of PDAC cells.

\section{Acknowledgements}

The authors would like to thank Dr Makiko Tsuboi, Ms. Miki Nishigawa, Ms. Rieko Takahashi and Mr. Shunichi Manabe for their technical assistance. The authors would also like to thank Dr Masahiko Sakaguchi for statistical analysis of the data.

\section{Funding}

The present study was supported by a Grant-in-Aid for Scientific Research (KAKENHI; grant nos. 24591013, 15K14396 and 17K09463).

\section{Availability of data and materials}

The datasets used and analyzed during the current study are available from the corresponding author on reasonable request.

\section{Authors' contributions}

KT conceived and designed the experiments. SN and KT performed the experiments. KT, MF and TS coordinated the research and analyzed the data. MF and SN performed pathological analyses. KT wrote the manuscript with contributions from all authors. TS supervised laboratorial processes. KT obtained financial support. All authors read and approved the final manuscript.

\section{Ethics approval and consent to participate}

The present study was approved by the Ethical Review Board of Kochi Medical School and Matsuyama Shimin Hospital (Nankoku, Japan) prior to patient recruitment. Written informed consent was acquired from each patient prior to initiation.

\section{Patient consent for publication}

Written informed consent was acquired from each patient prior to initiation.

\section{Competing interests}

The authors declare that they have no competing interests.

\section{References}

1. Kawasaki Y, Senda T, Ishidate T, Koyama R, Morishita T, Iwayama Y, Higuchi $\mathrm{O}$ and Akiyama T: Asef, a link between the tumor suppressor APC and G-protein signaling. Science 289: 1194-1197, 2000.
2. Thiesen S, Kübart S, Ropers HH and Nothwang HG: Isolation of two novel human RhoGEFs, ARHGEF3 and ARHGEF4, in 3p13-21 and 2q22. Biochem Biophys Res Commun 273: 364-369, 2000.

3. Kawasaki Y, Sato R and Akiyama T: Mutated APC and Asef are involved in the migration of colorectal tumour cells. Nat Cell Biol 5: 211-215, 2003.

4. Akiyama T and Kawasaki Y: Wnt signalling and the actin cytoskeleton. Oncogene 25: 7538-7544, 2006.

5. Mitin N, Betts L, Yohe ME, Der CJ, Sondek J and Rossman KL: Release of autoinhibition of ASEF by APC leads to CDC42 activation and tumor suppression. Nat Struct Mol Biol 14: 814-823, 2007.

6. Kawasaki Y, Tsuji S, Sagara M, Echizen K, Shibata Y and Akiyama T: Adenomatous polyposis coli and Asef function downstream of hepatocyte growth factor and phosphatidylinositol 3-kinase. J Biol Chem 284: 22436-22443, 2009.

7. Kawasaki Y, Tsuji S, Muroya K, Furukawa S, Shibata Y, Okuno M, Ohwada S and Akiyama T: The adenomatous polyposis coli-associated exchange factors Asef and Asef2 are required for adenoma formation in Apc(Min/+)mice. EMBO Rep 10: 1355-1362, 2009.

8. Kawasaki Y, Jigami T, Furukawa S, Sagara M,Echizen K, Shibata Y, Sato R and Akiyama T: The adenomatous polyposis coli-associated guanine nucleotide exchange factor Asef is involved in angiogenesis. J Biol Chem 285: 1199-1207, 2010.

9. DiMagno EP, Reber HA and Tempero MA; American Gastroenterological Association: AGA technical review on the epidemiology, diagnosis, and treatment of pancreatic ductal adenocarcinoma. Gastroenterology 117: 1464-1484, 1999.

10. Taniuchi K, Furihata M, Hanazaki K, Saito M and Saibara T: IGF2BP3-mediated translation in cell protrusions promotes cell invasiveness and metastasis of pancreatic cancer. Oncotarget 5: 6832-6845, 2014.

11. Taniuchi K, Furihata M and Saibara T: KIF20A-mediated RNA granule transport system promotes the invasiveness of pancreatic cancer cells. Neoplasia 16: 1082-1093, 2014.

12. Tsuboi M, Taniuchi K, Furihata M, Naganuma S, Kimura M, Watanabe R, Shimizu T, Saito M, Dabanaka K, Hanazaki K, et al: Vav3 is linked to poor prognosis of pancreatic cancers and promotes the motility and invasiveness of pancreatic cancer cells. Pancreatology 16: 905-916, 2016.

13. Japan Pancreatic Society: Classification of Pancreatic Carcinoma. 2nd English edition. Kanehara \& Co., Tokyo 2003.

14. Sobin LH, Gospodarowicz MK and Witteknd C: TNM classification of malignant tumors. 7th edition. Wiley-Blackwell, New York, NY, pp132-135, 2009.

15. Kondo S: Japanese pancreas society staging systems for pancreatic cancer. In Pancreatic Cancer. Springer, New York, NY, pp1035-1050, 2010.

16. Taniuchi K, Furihata M, Naganuma S, Dabanaka K, Hanazaki K and Saibara T: BCL7B, a predictor of poor prognosis of pancreatic cancers, promotes cell motility and invasion by influencing CREB signaling. Am J Cancer Res 8: 387-404, 2018.

17. Iwamura T, Katsuki $\mathrm{T}$ and Ide K: Establishment and characterization of a human pancreatic cancer cell line (SUIT-2) producing carcinoembryonic antigen and carbohydrate antigen 19-9. Jpn J Cancer Res 78: 54-62, 1987.

18. Taniuchi K, Nishimori I and Hollingsworth MA: Intracellular CD24 inhibits cell invasion by posttranscriptional regulation of BART through interaction with G3BP. Cancer Res 71: 895-905, 2011.

19. Taniuchi K, Furihata M, Naganuma S, Dabanaka K, Hanazaki K and Saibara T: PODXL, linked to poor prognosis of pancreatic cancers, promotes cell invasion via binding to gelsolin. Cancer Sci 107: 1430-1442, 2016.

20. Tanouchi A, Taniuchi K, Furihata M, Naganuma S, Dabanaka K, Kimura M, Watanabe R, Kohsaki T, Shimizu T, Saito M, et al: CCDC88A, a prognostic factor for human pancreatic cancers, promotes the motility and invasiveness of pancreatic cancer cells. J Exp Clin Cancer Res 35: 190, 2016.

21. Andea A, Sarkar F and Adsay VN: Clinicopathological correlates of pancreatic intraepithelial neoplasia: A comparative analysis of 82 cases with and 152 cases without pancreatic ductal adenocarcinoma. Mod Pathol 16: 996-1006, 2003.

22. Deer EL, González-Hernández J, Coursen JD, Shea JE, Ngatia J, Scaife CL, Firpo MA and Mulvihill SJ: Phenotype and genotype of pancreatic cancer cell lines. Pancreas 39: 425-435, 2010.

23. Taniuchi K, Furihata M, Iwasaki S, Tanaka K, Shimizu T, Saito $\mathrm{M}$ and Saibara T: RUVBL1 directly binds actin filaments and induces formation of cell protrusions to promote pancreatic cancer cell invasion. Int J Oncol 44: 1945-1954, 2014. 
24. Taniuchi K, Yokotani K and Saibara T: BART inhibits pancreatic cancer cell invasion by Racl inactivation through direct binding to active Rac1. Neoplasia 14: 440-450, 2012.

25. Yip-Schneider MT and Schmidt CM: MEK inhibition of pancreatic carcinoma cells by U0126 and its effect in combination with sulindac. Pancreas 27: 337-344, 2003.

26. Liao X, Zhang L, Thrasher JB, Du J and Li B: Glycogen synthase kinase-3beta suppression eliminates tumor necrosis factorrelated apoptosis-inducing ligand resistance in prostate cancer. Mol Cancer Ther 2: 1215-1222, 2003.

27. Min KJ, Han MA, Kim S, Park JW and Kwon TK: Osthole enhances TRAIL-mediated apoptosis through downregulation of c-FLIP expression in renal carcinoma Caki cells. Oncol Rep 37: 2348-2354, 2017

28. Ahrendt SA and Pitt HA: Surgical management of pancreatic cancer. Oncology (Williston Park) 16: 725-734, 736-738, 740 743, 2002.

29. Rescher U and Gerke V: Annexins - unique membrane binding proteins with diverse functions. J Cell Sci 117: 2631-2639, 2004.

30. Itoh RE, Kiyokawa E, Aoki K, Nishioka T, Akiyama T and Matsuda M: Phosphorylation and activation of the Rac1 and Cdc42 GEF Asef in A431 cells stimulated by EGF. J Cell Sci 121 2635-2642, 2008.

31. Farkas A, Szatmári E, Orbók A, Wilhelm I, Wejksza K, Nagyoszi P, Hutamekalin P, Bauer H, Bauer HC, Traweger A, et al: Hyperosmotic mannitol induces Src kinase-dependent phosphorylation of beta-catenin in cerebral endothelial cells. J Neurosci Res 80: 855-861, 2005.
32. Bowman T, Broome MA, Sinibaldi D, Wharton W, Pledger WJ, Sedivy JM, Irby R, Yeatman T, Courtneidge SA and Jove R: Stat3-mediated Myc expression is required for Src transformation and PDGF-induced mitogenesis. Proc Natl Acad Sci USA 98: 7319-7324, 2001

33. Levin VA: Basis and importance of Src as a target in cancer. Cancer Treat Res 119: 89-119, 2004.

34. He Y, Wang L, Liu W, Zhong J, Bai S, Wang Z, Thomas DG, Lin J, Reddy RM, Ramnath N, et al: MAP3K 3 expression in tumor cells and tumor-infiltrating lymphocytes is correlated with favorable patient survival in lung cancer. Sci Rep 5: 11471, 2015.

35. Taniuchi K, Furihata M, Hanazaki K, Iwasaki S, Tanaka K, Shimizu T, Saito M and Saibara T: Peroxiredoxin 1 promotes pancreatic cancer cell invasion by modulating p38 MAPK activity. Pancreas 44: 331-340, 2015.

36. Wilson W III and Baldwin AS: Maintenance of constitutive IkappaB kinase activity by glycogen synthase kinase-3alpha/ beta in pancreatic cancer. Cancer Res 68: 8156-8163, 2008.

37. Miyashita K, Nakada M, Shakoori A, Ishigaki Y, Shimasaki T, Motoo Y, Kawakami K and Minamoto T: An emerging strategy for cancer treatment targeting aberrant glycogen synthase kinase 3 beta. Anticancer Agents Med Chem 9: 1114-1122, 2009. 